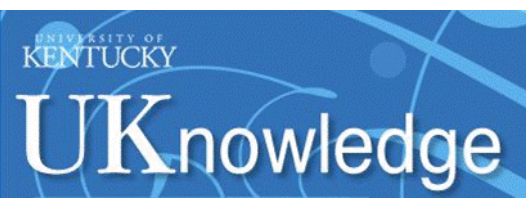

University of Kentucky

UKnowledge

$11-2014$

\title{
Comparison of Crystal Structures of 4-(benzo[b]thiophen-2-yl)-5-(3,4,5-trimethoxyphenyl)-2H-1,2,3-triazole and
} 4-(benzo[b]thiophen-2-yl)-2-methyl-5-(3,4,5-trimethoxyphenyl)-2H-1,2,3-triazo

Narsimha Reddy Penthala

University of Arkansas

Nikhil Reddy Madadi

University of Arkansas

Shobanbabu Bommagani

University of Arkansas

Sean Parkin

University of Kentucky, s.parkin@uky.edu

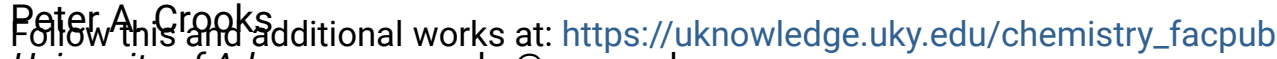
IIniversity of Arkansas, pacrooks@uams.edu

Part of the Chemistry Commons

Right click to open a feedback form in a new tab to let us know how this document benefits you.

\section{Repository Citation}

Penthala, Narsimha Reddy; Madadi, Nikhil Reddy; Bommagani, Shobanbabu; Parkin, Sean; and Crooks,

Peter A., "Comparison of Crystal Structures of

4-(benzo[b]thiophen-2-yl)-5-(3,4,5-trimethoxyphenyl)-2H-1,2,3-triazole and

4-(benzo[b]thiophen-2-yl)-2-methyl-5-(3,4,5-trimethoxyphenyl)-2H-1,2,3-triazole" (2014). Chemistry Faculty Publications. 28.

https://uknowledge.uky.edu/chemistry_facpub/28

This Article is brought to you for free and open access by the Chemistry at UKnowledge. It has been accepted for inclusion in Chemistry Faculty Publications by an authorized administrator of UKnowledge. For more information, please contact UKnowledge@lsv.uky.edu. 
Comparison of Crystal Structures of

4-(benzo[b]thiophen-2-yl)-5-(3,4,5-trimethoxyphenyl)-2H-1,2,3-triazole and 4-(benzo[b]thiophen-2-yl)-2-methyl-5-(3,4,5-trimethoxyphenyl)-2H-1,2,3-triazole

Digital Object Identifier (DOI)

https://doi.org/10.1107/S1600536814023095

Notes/Citation Information

Published in Acta Crystallographica Section E: Crystallographic Communications, v. 70, part 11, p. 392-395.

This is an open-access article distributed under the terms of the Creative Commons Attribution License, which permits unrestricted use, distribution, and reproduction in any medium, provided the original authors and source are cited.

This article is available at UKnowledge: https://uknowledge.uky.edu/chemistry_facpub/28 


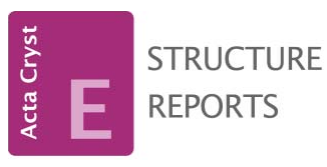

ISSN 1600-5368

Received 16 October 2014 Accepted 20 October 2014

Edited by P. C. Healy, Griffith University, Australia

Keywords: combretastatin A-4 analog; anticancer agent; triazole ring; hydrogen bonding; crystal structure

CCDC references: 1030172; 1030173 Supporting information: this article has supporting information at journals.iucr.org/e

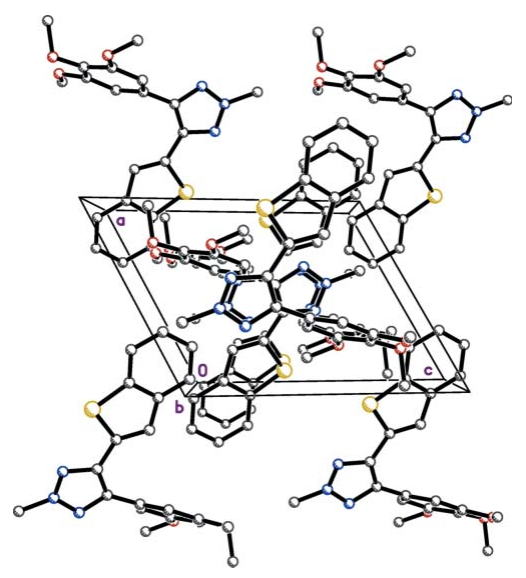

OPEN $\odot$ ACCESS
Comparison of crystal structures of 4-(benzo[b]thiophen-2-yl)-5-(3,4,5-trimethoxyphenyl)-2H1,2,3-triazole and 4-(benzo[b]thiophen-2-yl)-2methyl-5-(3,4,5-trimethoxyphenyl)-2H-1,2,3triazole

Narsimha Reddy Penthala, ${ }^{a}$ Nikhil Reddy Madadi, ${ }^{\text {a }}$ Shobanbabu Bommagani, ${ }^{a}$ Sean Parkin $^{\mathrm{b}}$ and Peter A. Crooks ${ }^{\mathrm{a} *}$

a Department of Pharmaceutical Sciences, College of Pharmacy, University of Arkansas for Medical Sciences, Little Rock, AR 72205, USA, and ${ }^{\mathbf{b}}$ Department of Chemistry, University of Kentucky, Lexington KY 40506, USA. *Correspondence e-mail: pacrooks@uams.edu

The title compound, $\mathrm{C}_{19} \mathrm{H}_{17} \mathrm{~N}_{3} \mathrm{O}_{3} \mathrm{~S}$ (I), was prepared by a [3+2]cycloaddition azide condensation reaction using sodium azide and L-proline as a Lewis base catalyst. $\mathrm{N}$-Methylation of compound (I) using $\mathrm{CH}_{3} \mathrm{I}$ gave compound (II), $\mathrm{C}_{20} \mathrm{H}_{19} \mathrm{~N}_{3} \mathrm{O}_{3} \mathrm{~S}$. The benzothiophene ring systems in (I) and (II) are almost planar, with r.m.s deviations from the mean plane $=0.0205$ (14) in (I) and 0.016 (2) $\AA$ in (II). In (I) and (II), the triazole rings make dihedral angles of 32.68 (5) and $10.43(8)^{\circ}$, respectively, with the mean planes of the benzothiophene ring systems. The trimethoxy phenyl rings make dihedral angles with the benzothiophene rings of 38.48 (4) in (I) and 60.43 (5) ${ }^{\circ}$ in (II). In the crystal of (I), the molecules are linked into chains by $\mathrm{N}-\mathrm{H} \cdots \mathrm{O}$ hydrogen bonds with $R_{1}^{2}(5)$ ring motifs. After the $N$-methylation of structure (I), no hydrogen-bonding interactions were observed for structure (II). The crystal structure of (II) has a minor component of disorder that corresponds to a $180^{\circ}$ flip of the benzothiophene ring system [occupancy ratio 0.9363 (14):0.0637 (14)].

\section{Chemical context}

In continuation of our work on the development of benzothiophene cyano combretastatin A-4 analogs as anti-cancer agents (Penthala et al., 2013), we have synthesized a series of novel CA-4 analogs by constructing a triazole ring structure (I) by chemical modification of the cyano group on the stilbene unit of cyano-CA-4 analogs utilizing a [3+2]cycloaddition azide condensation reaction with sodium azide in the presence of L-proline Lewis base as catalyst. This chemical modification is essential to restrict the tendency toward cis-trans isomerization of the cyano-stilbene moiety in cyano-CA-4 analogs (Penthala et al., 2013). To further check the position of the hydrogen atom in the triazole ring system in (I), an $\mathrm{N}$ methylation reaction was carried out on (I) using $\mathrm{CH}_{3} \mathrm{I}$, resulting in compound (II).

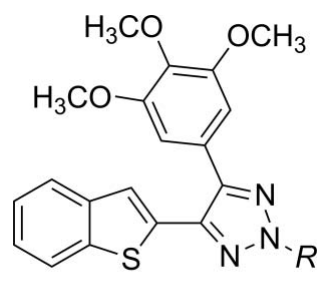

(I) $\mathrm{R}=\mathrm{H}$

(II) $\mathrm{R}=\mathrm{CH}_{3}$ 


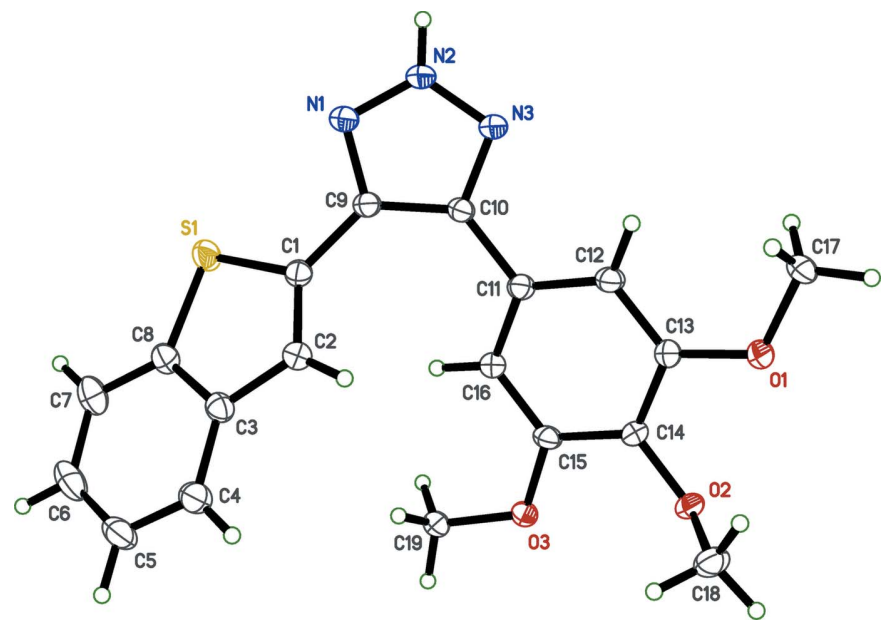

Figure 1

The molecular structure of (I), with displacement ellipsoids drawn at the $50 \%$ probability level.

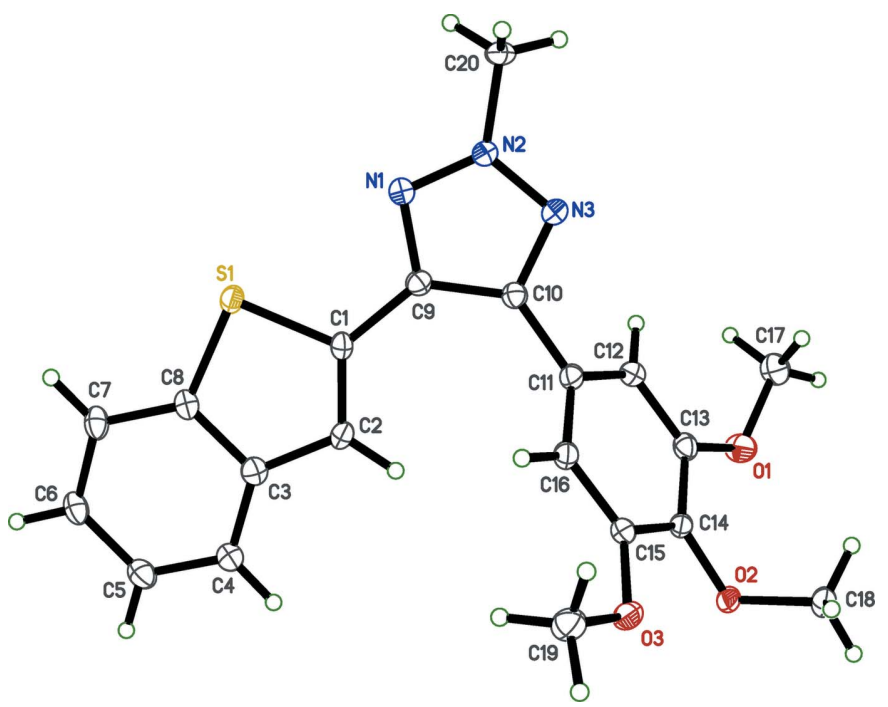

Figure 2

The molecular structure of (II), with displacement ellipsoids drawn at the $50 \%$ probability level.

\section{Structural commentary}

In order to obtain detailed information on the structural conformations of (I) and (II) for analysis of structure-activity relationships (SAR), including the position of the hydrogen atom in the triazole ring system of (I) and the position of methylation on the triazole ring system in (II), we determined the X-ray crystal structures of (I) and (II); see Figs. 1 and 2, respectively.

Selected geometric parameters are given in Tables 1 and 2 for (I) and (II), respectively. The benzothiophene rings are almost planar with r.m.s deviations from the mean plane of 0.0205 (14) in (I) and 0.016 (2) $\AA$ in (II), with bond distances and angles comparable with those reported for other benzothiophene derivatives (Sonar et al., 2007) and triazole analogs (Madadi et al., 2014). The triazole rings make dihedral angles of $32.68(5)^{\circ}$ and $10.43(8)^{\circ}$, respectively, in (I) and (II) with the mean plane of the benzothiophene ring systems. The tri-
Table 1

Selected geometric parameters $\left(\AA,^{\circ}\right)$ for $(\mathrm{I})$.

\begin{tabular}{lcll}
\hline $\mathrm{N} 1-\mathrm{N} 2$ & $1.324(2)$ & $\mathrm{N} 2-\mathrm{H} 2 N$ & $0.87(2)$ \\
$\mathrm{N} 1-\mathrm{C} 9$ & $1.343(2)$ & $\mathrm{N} 3-\mathrm{C} 10$ & $1.345(2)$ \\
$\mathrm{N} 2-\mathrm{N} 3$ & $1.330(2)$ & & \\
& & & \\
$\mathrm{C} 8-\mathrm{S} 1-\mathrm{C} 1$ & $91.50(8)$ & $\mathrm{C} 10-\mathrm{C} 9-\mathrm{C} 1$ & $131.64(14)$ \\
$\mathrm{N} 2-\mathrm{N} 1-\mathrm{C} 9$ & $103.74(13)$ & $\mathrm{C} 9-\mathrm{C} 10-\mathrm{C} 11$ & $131.16(14)$ \\
$\mathrm{N} 2-\mathrm{N} 3-\mathrm{C} 10$ & $103.74(13)$ & $\mathrm{O} 1-\mathrm{C} 13-\mathrm{C} 14$ & $114.89(14)$ \\
$\mathrm{C} 4-\mathrm{C} 3-\mathrm{C} 2$ & $129.50(16)$ & & \\
\hline
\end{tabular}

Table 2

Selected geometric parameters $\left(\AA{ }^{\circ},{ }^{\circ}\right)$ for (II).

\begin{tabular}{lclc}
\hline $\mathrm{N} 1-\mathrm{N} 2$ & $1.3266(15)$ & $\mathrm{N} 2-\mathrm{C} 20$ & $1.4527(16)$ \\
$\mathrm{N} 1-\mathrm{C} 9$ & $1.3477(16)$ & $\mathrm{N} 3-\mathrm{C} 10$ & $1.3450(16)$ \\
$\mathrm{N} 2-\mathrm{N} 3$ & $1.3279(15)$ & & \\
& & & \\
$\mathrm{N} 1-\mathrm{N} 2-\mathrm{N} 3$ & $115.92(10)$ & $\mathrm{C} 4^{\prime}-\mathrm{C} 3^{\prime}-\mathrm{C} 2^{\prime}$ & $132(2)$ \\
$\mathrm{C} 2-\mathrm{C} 1-\mathrm{C} 9$ & $129.94(17)$ & $\mathrm{C} 7^{\prime}-\mathrm{C}^{\prime}-\mathrm{S} 1^{\prime}$ & $129(2)$ \\
$\mathrm{C} 8-\mathrm{S} 1-\mathrm{C} 1$ & $91.33(8)$ & $\mathrm{C}^{\prime}-\mathrm{C} 9-\mathrm{C} 10$ & $127.3(11)$ \\
$\mathrm{C} 4-\mathrm{C} 3-\mathrm{C} 2$ & $129.79(17)$ & $\mathrm{C} 10-\mathrm{C} 9-\mathrm{C} 1$ & $132.41(13)$ \\
$\mathrm{C} 9-\mathrm{C} 1^{\prime}-\mathrm{S} 1^{\prime}$ & $128.0(18)$ & $\mathrm{C} 9-\mathrm{C} 10-\mathrm{C} 11$ & $132.90(12)$ \\
$\mathrm{C} 8^{\prime}-\mathrm{S}^{\prime}-\mathrm{C} 1^{\prime}$ & $95.8(12)$ & & \\
\hline
\end{tabular}

methoxyphenyl rings make dihedral angles of 38.48 (4) in (I) and $60.43(5)^{\circ}$ in (II) with the benzothiophene ring systems. In both compounds (I) and (II), deviations from ideal geometry are observed in the bond angles $\mathrm{C} 1-\mathrm{S} 1-\mathrm{C} 8, \mathrm{~N} 2-\mathrm{N} 1-\mathrm{C} 9$, $\mathrm{N} 2-\mathrm{N} 3-\mathrm{C} 10$, which are compressed, and $\mathrm{C} 1-\mathrm{C} 9-\mathrm{C} 10$, $\mathrm{C} 9-\mathrm{C} 10-\mathrm{C} 11, \mathrm{C} 2-\mathrm{C} 3-\mathrm{C} 4$, which are expanded (see Tables 1 and 2). After $N$-methylation, no significant difference is observed for the $\mathrm{N} 1-\mathrm{N} 2-\mathrm{N} 3$ bond angle [116.2 (1) and $115.9(1)^{\circ}$, respectively, for (I) and (II)]. The crystal structure of (II) has a minor component of disorder that corresponds to a $180^{\circ}$ flip of the benzothiophene ring system [occupancy ratio 0.9363 (14):0.0637 (14)].

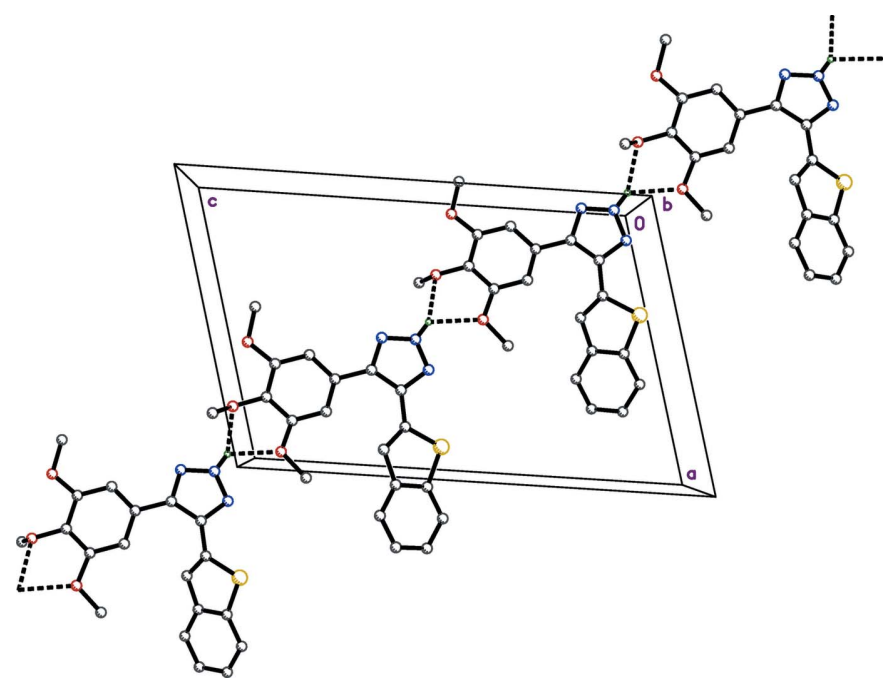

Figure 3

Hydrogen bonding in the crystal structure of (I), viewed along the $b$ axis. Dashed lines represent hydrogen bonds, which join molecules into chains along the [101] direction. 
Table 3

Hydrogen-bond geometry $\left(\AA,^{\circ}\right)$ for (I).

\begin{tabular}{lllll}
\hline$D-\mathrm{H} \cdots A$ & $D-\mathrm{H}$ & $\mathrm{H} \cdots A$ & $D \cdots A$ & $D-\mathrm{H} \cdots A$ \\
\hline $\mathrm{N} 2-\mathrm{H} 2 N \cdots \mathrm{O} 2^{\mathrm{i}}$ & $0.87(2)$ & $2.16(2)$ & $2.9381(18)$ & $147.6(18)$ \\
$\mathrm{N} 2-\mathrm{H} 2 N \cdots{ }^{\mathrm{i}}{ }^{\mathrm{i}}$ & $0.87(2)$ & $2.20(2)$ & $2.8503(18)$ & $130.8(17)$ \\
\hline
\end{tabular}

Symmetry code: (i) $x-\frac{1}{2},-y+\frac{3}{2}, z-\frac{1}{2}$.

\section{Supramolecular features}

Hydrogen bonding and the mode of packing of (I) is illustrated in Fig. 3, and the mode of packing of (II) is illustrated in Fig. 4. In the structure of (I), the molecules are linked by intermolecular hydrogen bonds $(\mathrm{N} 2-\mathrm{H} 2 \mathrm{~N} \cdots \mathrm{O} 2$ and $\mathrm{N} 2-$ $\mathrm{H} 2 N \cdots \mathrm{O} 3$ ), forming $R_{1}^{2}(5)$ ring motifs (Table 3), which propagate as chains along the [101] direction. Contacts between adjacent chains form two-dimensional pleated-sheet networks in the $a c$ plane. No significant hydrogen-bonding interactions were found in the structure of (II).

\section{Database survey}

A search of the 2014 release of the Cambridge Structural Database on unit-cell dimensions for (I) and (II) revealed four triazole structures (HOZZAY, UPEWAO, SAFZEG \& VUSNEC), although none bore any particular relation to compounds (I) or (II). A search on the triazole ring fragment with either $\mathrm{H}$ or methyl attached to the middle $\mathrm{N}$ atom

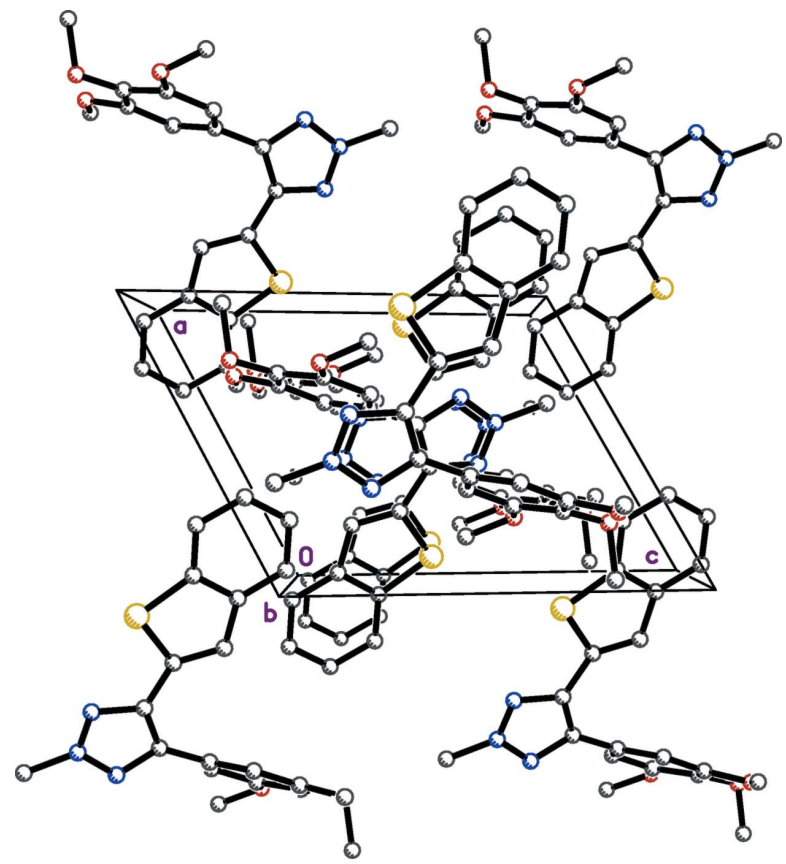

Figure 4

Crystal packing of (II), as viewed along the $b$ axis.

revealed 48 and 17 hits, respectively, none of which contained either benzothiophene or trimethoxybenzene functional groups.

Table 4

Experimental details.

(I)

Crystal data

Chemical formula

$M_{\mathrm{r}}$

Crystal system, space group

Temperature (K)

$a, b, c(\AA)$

$\alpha, \beta, \gamma\left({ }^{\circ}\right)$

$V\left(\AA^{3}\right)$

$Z$

Radiation type

$\mu\left(\mathrm{mm}^{-1}\right)$

Crystal size (mm)

Data collection

Diffractometer

Absorption correction

$T_{\min }, T_{\max }$

No. of measured, independent and observed

$[I>2 \sigma(I)]$ reflections

$R_{\text {int }}$

$(\sin \theta / \lambda)_{\max }\left(\AA^{-1}\right)$

Refinement

$R\left[F^{2}>2 \sigma\left(F^{2}\right)\right], w R\left(F^{2}\right), S$

No. of reflections

No. of parameters

No. of restraints

$\mathrm{H}$-atom treatment

$\Delta \rho_{\max }, \Delta \rho_{\min }\left(\mathrm{e} \AA^{-3}\right)$

(II)

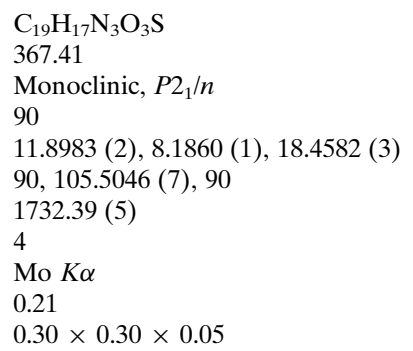

Nonius KappaCCD

Multi-scan (SADABS; Sheldrick, 2008a)

$0.816,0.966$

28105, 3984, 3093

0.045

0.650

$0.044,0.124,1.07$

3984

241

0

$\mathrm{H}$ atoms treated by a mixture of independent and constrained refinement

$0.55,-0.29$

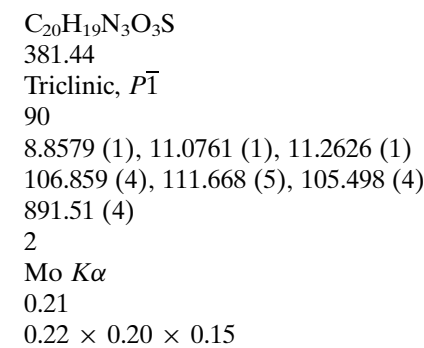

Nonius KappaCCD

Multi-scan (SADABS; Sheldrick, 2008a)

$0.858,0.962$

$36591,4097,3572$

0.045

0.651

$0.037,0.096,1.08$

4097

276

161

H-atom parameters constrained

$0.31,-0.28$ 


\section{Synthesis and crystallization}

The title compounds were prepared according to a previously reported procedure (Penthala et al., 2014). Recrystallization from methanol afforded (I) and (II) as yellow and pale-yellow crystalline products, respectively, which were suitable for $\mathrm{X}$-ray analysis.

\section{Refinement details}

Crystal data, data collection and structure refinement details are summarized in Table $4 . \mathrm{H}$ atoms were found in difference Fourier maps. Carbon-bound hydrogens were subsequently placed at idealized positions with constrained distances of 0.98 $\left(R \mathrm{CH}_{3}\right)$ and $0.95 \AA\left(\mathrm{Csp}^{2} \mathrm{H}\right)$. Coordinates of the $\mathrm{N}$-bound hydrogen were refined freely. $U_{\text {iso }}(\mathrm{H})$ values were set to either $1.2 U_{\text {eq }}$ or $1.5 U_{\text {eq }}\left(R \mathrm{CH}_{3}\right)$ of the attached atom.

Refinement progress was checked using PLATON (Spek, 2009 ) and by an $R$-tensor (Parkin, 2000). To ensure satisfactory refinement of disordered groups in the structure, a combination of constraints and restraints was employed. The constraints (SHELXL command EADP) were used to fix overlapping fragments. Restraints were used to maintain the integrity of ill-defined or disordered groups (SHELXL commands SAME and RIGU).

In structure (II), there was a small amount of a second conformation for the benzothiophene ring systems, with major and minor component fractions of 93.63 (14) and 6.37 (14)\%, respectively.

\section{Acknowledgements}

This investigation was supported by NIH/National Cancer Institute grant R01 CA140409.

\section{References}

Madadi, N. R., Penthala, N. R., Bommagani, S., Parkin, S. \& Crooks, P. A. (2014). Acta Cryst. E70, o1128-o1129.

Nonius (1998). Collect Nonius BV, Delft, The Netherlands.

Otwinowski, Z. \& Minor, W. (2006). International Tables for Crystallography, Vol. F, ch. 11.4, pp. 226-235. Dordrecht: Kluwer Academic Publishers.

Parkin, S. (2000). Acta Cryst. A56, 157-162.

Parkin, S. (2013). CIFFIX. http://xray.uky.edu/people/parkin/ programs/ciffix

Penthala, N. R., Madadi, N. R., Janganati, V. \& Crooks, P. A. (2014). Tetrahedron Lett. 55, 5562-5565.

Penthala, N. R., Sonar, V. N., Horn, J., Leggas, M., Yadlapalli, K. B. J. S. \& Crooks, P. A. (2013). Med. Chem. Commun. 4, 10731078.

Sheldrick, G. M. (2008a). SADABS. University of Göttingen, Germany.

Sheldrick, G. M. (2008b). Acta Cryst. A64, 112-122.

Sonar, V. N., Parkin, S. \& Crooks, P. A. (2007). Acta Cryst. C63, o7430745.

Spek, A. L. (2009). Acta Cryst. D65, 148-155. 


\section{supporting information}

Acta Cryst. (2014). E70, 392-395 [doi:10.1107/S1600536814023095]

Comparison of crystal structures of 4-(benzo[b]thiophen-2-yl)-5-(3,4,5-trimethoxyphenyl)-2H-1,2,3-triazole and 4-(benzo[b]thiophen-2-yl)-2-methyl-5-(3,4,5trimethoxyphenyl)-2H-1,2,3-triazole

Narsimha Reddy Penthala, Nikhil Reddy Madadi, Shobanbabu Bommagani, Sean Parkin and

Peter A. Crooks

\section{Computing details}

For both compounds, data collection: COLLECT (Nonius, 1998); cell refinement: SCALEPACK (Otwinowski \& Minor, 2006); data reduction: DENZO-SMN (Otwinowski \& Minor, 2006); program(s) used to solve structure: SHELXS97

(Sheldrick, 2008b). Program(s) used to refine structure: SHELXL2013 (Sheldrick, 2008b) for (I); SHELXL2014

(Sheldrick, 2008b) for (II). For both compounds, molecular graphics: XP in SHELXTL (Sheldrick, 2008b). Software used to prepare material for publication: SHELXL2013 (Sheldrick, 2008b) and CIFFIX (Parkin, 2013) for (I); SHELXL2014 (Sheldrick, 2008b) and CIFFIX (Parkin, 2013) for (II).

(I) 4-(Benzo[b]thiophen-2-yl)-5-(3,4,5-trimethoxyphenyl)-2H-1,2,3-triazole

Crystal data

$\mathrm{C}_{19} \mathrm{H}_{17} \mathrm{~N}_{3} \mathrm{O}_{3} \mathrm{~S}$

$M_{r}=367.41$

Monoclinic, $P 22_{1} / n$

$a=11.8983(2) \AA$

$b=8.1860(1) \AA$

$c=18.4582(3) \AA$

$\beta=105.5046(7)^{\circ}$

$V=1732.39(5) \AA^{3}$

$Z=4$

Data collection

\section{Nonius KappaCCD} diffractometer

Radiation source: fine-focus sealed-tube Detector resolution: 9.1 pixels $\mathrm{mm}^{-1}$ $\varphi$ and $\omega$ scans at fixed $\chi=55^{\circ}$

Absorption correction: multi-scan (SADABS; Sheldrick, 2008a)

$T_{\min }=0.816, T_{\max }=0.966$
$F(000)=768$

$D_{\mathrm{x}}=1.409 \mathrm{Mg} \mathrm{m}^{-3}$

Mo $K \alpha$ radiation, $\lambda=0.71073 \AA$

Cell parameters from 4236 reflections

$\theta=1.0-27.5^{\circ}$

$\mu=0.21 \mathrm{~mm}^{-1}$

$T=90 \mathrm{~K}$

Plate, yellow

$0.30 \times 0.30 \times 0.05 \mathrm{~mm}$

28105 measured reflections 3984 independent reflections 3093 reflections with $I>2 \sigma(I)$

$R_{\text {int }}=0.045$

$\theta_{\text {max }}=27.5^{\circ}, \theta_{\text {min }}=1.8^{\circ}$

$h=-15 \rightarrow 15$

$k=-10 \rightarrow 10$

$l=-23 \rightarrow 22$ 


\section{Refinement}

Refinement on $F^{2}$

Least-squares matrix: full

$R\left[F^{2}>2 \sigma\left(F^{2}\right)\right]=0.044$

$w R\left(F^{2}\right)=0.124$

$S=1.07$

3984 reflections

241 parameters

0 restraints

Primary atom site location: structure-invariant direct methods
Secondary atom site location: difference Fourier map

Hydrogen site location: difference Fourier map

$\mathrm{H}$ atoms treated by a mixture of independent and constrained refinement

$w=1 /\left[\sigma^{2}\left(F_{\mathrm{o}}^{2}\right)+(0.0707 P)^{2}+0.6092 P\right]$ where $P=\left(F_{\mathrm{o}}^{2}+2 F_{\mathrm{c}}^{2}\right) / 3$

$(\Delta / \sigma)_{\max }<0.001$

$\Delta \rho_{\max }=0.55 \mathrm{e}^{-3}$

$\Delta \rho_{\min }=-0.29$ e $\AA^{-3}$

Special details

Experimental. The crystal was mounted with polyisobutene oil on the tip of a fine glass fibre, fastened in a copper mounting pin with electrical solder. It was placed directly into the cold stream of a liquid nitrogen based cryostat, according to published methods (Hope, H. (1994). Prog. Inorg. Chem. 41, 1-19; Parkin, S. \& Hope, H. (1998). J. Appl. Cryst. 31, 945-953.).

Diffraction data were collected with the crystal at $90 \mathrm{~K}$, which is standard practice in this laboratory for the majority of flash-cooled crystals.

Geometry. All e.s.d.'s (except the e.s.d. in the dihedral angle between two 1.s. planes) are estimated using the full covariance matrix. The cell e.s.d.'s are taken into account individually in the estimation of e.s.d.'s in distances, angles and torsion angles; correlations between e.s.d.'s in cell parameters are only used when they are defined by crystal symmetry. An approximate (isotropic) treatment of cell e.s.d.'s is used for estimating e.s.d.'s involving l.s. planes.

Refinement. Refinement progress was checked using PLATON (Spek, 2009) and by an R-tensor (Parkin, 2000). The final model was further checked with the IUCr utility checkCIF.

Fractional atomic coordinates and isotropic or equivalent isotropic displacement parameters $\left(\hat{A}^{2}\right)$

\begin{tabular}{|c|c|c|c|c|}
\hline & $x$ & $y$ & $z$ & $U_{\text {iso }} * / U_{\text {eq }}$ \\
\hline $\mathrm{S} 1$ & $0.39424(4)$ & $0.67530(6)$ & 0.05935 (2) & $0.02500(14)$ \\
\hline N1 & $0.13369(12)$ & $0.64131(18)$ & $0.05222(8)$ & 0.0209 (3) \\
\hline N2 & $0.03228(12)$ & $0.67054(18)$ & $0.06649(8)$ & $0.0214(3)$ \\
\hline $\mathrm{H} 2 \mathrm{~N}$ & $-0.0306(18)$ & $0.686(2)$ & $0.0298(12)$ & $0.026^{*}$ \\
\hline N3 & $0.03376(12)$ & $0.68041(18)$ & $0.13868(8)$ & 0.0201 \\
\hline $\mathrm{O} 1$ & $0.07856(10)$ & $0.60706(15)$ & $0.42702(6)$ & 0.0223 \\
\hline $\mathrm{O} 2$ & $0.30089(10)$ & $0.69738(14)$ & $0.49128(6)$ & 0.0196 \\
\hline $\mathrm{O} 3$ & $0.44759(9)$ & $0.76268(15)$ & 0.40977 (6) & 0.0208 \\
\hline $\mathrm{C} 1$ & $0.33215(14)$ & $0.5986(2)$ & $0.12815(9)$ & $0.0186(3)$ \\
\hline $\mathrm{C} 2$ & $0.41132(14)$ & $0.5191(2)$ & $0.18353(9)$ & 0.0195 \\
\hline H2 & 0.3920 & 0.4676 & 0.2247 & $0.023^{*}$ \\
\hline $\mathrm{C} 3$ & $0.52685(14)$ & $0.5204(2)$ & $0.17387(9)$ & $0.0200(4)$ \\
\hline $\mathrm{C} 4$ & $0.63017(15)$ & $0.4549(2)$ & $0.22005(10)$ & $0.0268(4)$ \\
\hline $\mathrm{H} 4$ & 0.6291 & 0.3961 & 0.2642 & $0.032 *$ \\
\hline $\mathrm{C} 5$ & $0.73349(15)$ & $0.4767(2)$ & $0.20067(11)$ & $0.0307(4)$ \\
\hline H5 & 0.8037 & 0.4322 & 0.2317 & $0.037^{*}$ \\
\hline C6 & $0.73606(16)$ & 0.5633 & $0.13592(12)$ & $0.0331(5)$ \\
\hline H6 & 0.8084 & 0.5787 & 0.1242 & $0.040^{*}$ \\
\hline $\mathrm{C} 7$ & $0.63591(16)$ & $0.6268(2)$ & $0.08883(12)$ & $0.0299(4)$ \\
\hline $\mathrm{H7}$ & 0.6380 & 0.6841 & 0.0445 & $0.036^{*}$ \\
\hline $\mathrm{C} 8$ & $0.53112(14)$ & $0.6047(2)$ & $0.10787(10)$ & $0.0217(4)$ \\
\hline
\end{tabular}




$\begin{array}{lllll}\text { C9 } & 0.20875(14) & 0.6295(2) & 0.12074(9) & 0.0173(3) \\ \text { C10 } & 0.14626(13) & 0.6547(2) & 0.17521(9) & 0.0169(3) \\ \text { C11 } & 0.18559(14) & 0.6599(2) & 0.25781(9) & 0.0171(3) \\ \text { C12 } & 0.10917(14) & 0.6200(2) & 0.30137(9) & 0.0185(3) \\ \text { H12 } & 0.0323 & 0.5837 & 0.2778 & 0.022^{*} \\ \text { C13 } & 0.14657(14) & 0.6340(2) & 0.37917(9) & 0.0181(3) \\ \text { C14 } & 0.26154(14) & 0.6801(2) & 0.41410(9) & 0.0171(3) \\ \text { C15 } & 0.33703(13) & 0.7182(2) & 0.37040(9) & 0.0176(3) \\ \text { C16 } & 0.29866(14) & 0.7127(2) & 0.29248(9) & 0.0172(3) \\ \text { H16 } & 0.3492 & 0.7448 & 0.2629 & 0.021^{*} \\ \text { C17 } & -0.04088(14) & 0.5647(3) & 0.39398(10) & 0.0260(4) \\ \text { H17A } & -0.0797 & 0.6537 & 0.3612 & 0.039^{*} \\ \text { H17B } & -0.0800 & 0.5468 & 0.4338 & 0.039^{*} \\ \text { H17C } & -0.0447 & 0.4646 & 0.3643 & 0.039^{*} \\ \text { C18 } & 0.32569(16) & 0.5438(2) & 0.53021(10) & 0.0259(4) \\ \text { H18A } & 0.2566 & 0.4735 & 0.5159 & 0.039^{*} \\ \text { H18B } & 0.3464 & 0.5630 & 0.5846 & 0.039^{*} \\ \text { H18C } & 0.3909 & 0.4904 & 0.5167 & 0.039^{*} \\ \text { C19 } & 0.53268(14) & 0.7777(2) & 0.36776(9) & 0.0212(4) \\ \text { H19A } & 0.5353 & 0.6761 & 0.3402 & 0.032^{*} \\ \text { H19B } & 0.6095 & 0.7987 & 0.4023 & 0.032^{*} \\ \text { H19C } & 0.5113 & 0.8685 & 0.3321 & 0.032^{*}\end{array}$

Atomic displacement parameters $\left(\AA^{2}\right)$

\begin{tabular}{lllllll}
\hline & $U^{11}$ & $U^{22}$ & $U^{33}$ & $U^{12}$ & $U^{13}$ & $U^{23}$ \\
\hline $\mathrm{S} 1$ & $0.0206(2)$ & $0.0338(3)$ & $0.0226(2)$ & $0.00439(18)$ & $0.00931(17)$ & $0.00745(18)$ \\
$\mathrm{N} 1$ & $0.0158(7)$ & $0.0285(8)$ & $0.0174(7)$ & $-0.0002(6)$ & $0.0024(5)$ & $0.0010(6)$ \\
$\mathrm{N} 2$ & $0.0157(7)$ & $0.0307(8)$ & $0.0159(7)$ & $0.0012(6)$ & $0.0006(6)$ & $0.0012(6)$ \\
$\mathrm{N} 3$ & $0.0156(7)$ & $0.0280(8)$ & $0.0155(7)$ & $-0.0002(6)$ & $0.0018(5)$ & $0.0006(6)$ \\
O1 & $0.0161(6)$ & $0.0335(7)$ & $0.0177(6)$ & $-0.0042(5)$ & $0.0052(5)$ & $0.0008(5)$ \\
O2 & $0.0195(6)$ & $0.0246(6)$ & $0.0131(5)$ & $-0.0010(5)$ & $0.0018(4)$ & $0.0004(5)$ \\
O3 & $0.0130(5)$ & $0.0316(7)$ & $0.0172(6)$ & $-0.0039(5)$ & $0.0028(4)$ & $-0.0021(5)$ \\
C1 & $0.0164(8)$ & $0.0221(9)$ & $0.0172(8)$ & $-0.0025(6)$ & $0.0043(6)$ & $-0.0024(6)$ \\
C2 & $0.0180(8)$ & $0.0209(9)$ & $0.0192(8)$ & $0.0007(7)$ & $0.0043(6)$ & $0.0000(6)$ \\
C3 & $0.0174(8)$ & $0.0200(9)$ & $0.0228(8)$ & $-0.0011(6)$ & $0.0054(6)$ & $-0.0037(7)$ \\
C4 & $0.0222(9)$ & $0.0298(10)$ & $0.0265(9)$ & $0.0032(7)$ & $0.0033(7)$ & $-0.0050(8)$ \\
C5 & $0.0172(8)$ & $0.0348(11)$ & $0.0370(10)$ & $0.0046(8)$ & $0.0021(7)$ & $-0.0094(9)$ \\
C6 & $0.0187(9)$ & $0.0350(11)$ & $0.0487(12)$ & $-0.0004(8)$ & $0.0143(8)$ & $-0.0058(9)$ \\
C7 & $0.0246(9)$ & $0.0310(10)$ & $0.0389(11)$ & $-0.0005(8)$ & $0.0169(8)$ & $0.0014(8)$ \\
C8 & $0.0189(8)$ & $0.0234(9)$ & $0.0239(9)$ & $0.0007(7)$ & $0.0076(7)$ & $0.0002(7)$ \\
C9 & $0.0157(8)$ & $0.0197(8)$ & $0.0156(8)$ & $-0.0001(6)$ & $0.0025(6)$ & $0.0009(6)$ \\
C10 & $0.0134(7)$ & $0.0201(8)$ & $0.0164(8)$ & $-0.0004(6)$ & $0.0025(6)$ & $0.0011(6)$ \\
C11 & $0.0160(8)$ & $0.0181(8)$ & $0.0165(8)$ & $0.0012(6)$ & $0.0030(6)$ & $-0.0001(6)$ \\
C12 & $0.0155(8)$ & $0.0211(9)$ & $0.0174(8)$ & $-0.0014(6)$ & $0.0016(6)$ & $0.0001(6)$ \\
C13 & $0.0168(8)$ & $0.0211(9)$ & $0.0170(8)$ & $-0.0007(6)$ & $0.0055(6)$ & $0.0014(6)$ \\
C14 & $0.0176(8)$ & $0.0197(8)$ & $0.0132(7)$ & $0.0008(6)$ & $0.0028(6)$ & $0.0011(6)$ \\
C15 & $0.0129(7)$ & $0.0196(8)$ & $0.0183(8)$ & $-0.0004(6)$ & $0.0007(6)$ & $-0.0021(6)$ \\
& & & & &
\end{tabular}


supporting information

\begin{tabular}{lllllll} 
C16 & $0.0159(8)$ & $0.0196(8)$ & $0.0160(8)$ & $0.0006(6)$ & $0.0037(6)$ & $0.0004(6)$ \\
C17 & $0.0139(8)$ & $0.0400(11)$ & $0.0235(9)$ & $-0.0043(7)$ & $0.0041(7)$ & $0.0044(8)$ \\
C18 & $0.0266(9)$ & $0.0292(10)$ & $0.0197(8)$ & $-0.0005(7)$ & $0.0024(7)$ & $0.0069(7)$ \\
C19 & $0.0143(8)$ & $0.0301(9)$ & $0.0193(8)$ & $-0.0014(7)$ & $0.0047(6)$ & $0.0006(7)$ \\
\hline
\end{tabular}

Geometric parameters $\left(A,{ }^{\circ}\right)$

\begin{tabular}{|c|c|c|c|}
\hline $\mathrm{S} 1-\mathrm{C} 8$ & $1.7345(17)$ & $\mathrm{C} 6-\mathrm{C} 7$ & $1.375(3)$ \\
\hline $\mathrm{S} 1-\mathrm{C} 1$ & $1.7474(17)$ & C6- $\mathrm{H} 6$ & 0.9500 \\
\hline $\mathrm{N} 1-\mathrm{N} 2$ & $1.324(2)$ & $\mathrm{C} 7-\mathrm{C} 8$ & $1.395(2)$ \\
\hline $\mathrm{N} 1-\mathrm{C} 9$ & $1.343(2)$ & $\mathrm{C} 7-\mathrm{H} 7$ & 0.9500 \\
\hline $\mathrm{N} 2-\mathrm{N} 3$ & $1.330(2)$ & $\mathrm{C} 9-\mathrm{C} 10$ & $1.416(2)$ \\
\hline $\mathrm{N} 2-\mathrm{H} 2 \mathrm{~N}$ & $0.87(2)$ & $\mathrm{C} 10-\mathrm{C} 11$ & $1.471(2)$ \\
\hline $\mathrm{N} 3-\mathrm{C} 10$ & $1.345(2)$ & $\mathrm{C} 11-\mathrm{C} 16$ & $1.395(2)$ \\
\hline $\mathrm{O} 1-\mathrm{C} 13$ & $1.3660(19)$ & $\mathrm{C} 11-\mathrm{C} 12$ & $1.404(2)$ \\
\hline $\mathrm{O} 1-\mathrm{C} 17$ & $1.4313(19)$ & $\mathrm{C} 12-\mathrm{C} 13$ & $1.390(2)$ \\
\hline $\mathrm{O} 2-\mathrm{C} 14$ & $1.3829(19)$ & $\mathrm{C} 12-\mathrm{H} 12$ & 0.9500 \\
\hline $\mathrm{O} 2-\mathrm{C} 18$ & $1.439(2)$ & $\mathrm{C} 13-\mathrm{C} 14$ & $1.399(2)$ \\
\hline $\mathrm{O} 3-\mathrm{C} 15$ & $1.3712(19)$ & $\mathrm{C} 14-\mathrm{C} 15$ & $1.394(2)$ \\
\hline $\mathrm{O} 3-\mathrm{C} 19$ & $1.4356(19)$ & $\mathrm{C} 15-\mathrm{C} 16$ & $1.389(2)$ \\
\hline $\mathrm{C} 1-\mathrm{C} 2$ & $1.357(2)$ & C16-H16 & 0.9500 \\
\hline $\mathrm{C} 1-\mathrm{C} 9$ & $1.460(2)$ & C17-H17A & 0.9800 \\
\hline $\mathrm{C} 2-\mathrm{C} 3$ & $1.433(2)$ & C17-H17B & 0.9800 \\
\hline $\mathrm{C} 2-\mathrm{H} 2$ & 0.9500 & $\mathrm{C} 17-\mathrm{H} 17 \mathrm{C}$ & 0.9800 \\
\hline $\mathrm{C} 3-\mathrm{C} 4$ & $1.402(2)$ & C18-H18A & 0.9800 \\
\hline $\mathrm{C} 3-\mathrm{C} 8$ & $1.413(2)$ & $\mathrm{C} 18-\mathrm{H} 18 \mathrm{~B}$ & 0.9800 \\
\hline $\mathrm{C} 4-\mathrm{C} 5$ & $1.381(3)$ & $\mathrm{C} 18-\mathrm{H} 18 \mathrm{C}$ & 0.9800 \\
\hline $\mathrm{C} 4-\mathrm{H} 4$ & 0.9500 & C19-H19A & 0.9800 \\
\hline $\mathrm{C} 5-\mathrm{C} 6$ & $1.397(3)$ & C19-H19B & 0.9800 \\
\hline $\mathrm{C} 5-\mathrm{H} 5$ & 0.9500 & $\mathrm{C} 19-\mathrm{H} 19 \mathrm{C}$ & 0.9800 \\
\hline $\mathrm{C} 8-\mathrm{S} 1-\mathrm{C} 1$ & $91.50(8)$ & $\mathrm{C} 9-\mathrm{C} 10-\mathrm{C} 11$ & $131.16(14)$ \\
\hline $\mathrm{N} 2-\mathrm{N} 1-\mathrm{C} 9$ & $103.74(13)$ & $\mathrm{C} 16-\mathrm{C} 11-\mathrm{C} 12$ & $120.15(15)$ \\
\hline $\mathrm{N} 1-\mathrm{N} 2-\mathrm{N} 3$ & $116.21(14)$ & $\mathrm{C} 16-\mathrm{C} 11-\mathrm{C} 10$ & $119.01(14)$ \\
\hline $\mathrm{N} 1-\mathrm{N} 2-\mathrm{H} 2 \mathrm{~N}$ & $120.6(13)$ & $\mathrm{C} 12-\mathrm{C} 11-\mathrm{C} 10$ & $120.77(14)$ \\
\hline $\mathrm{N} 3-\mathrm{N} 2-\mathrm{H} 2 \mathrm{~N}$ & $123.2(13)$ & $\mathrm{C} 13-\mathrm{C} 12-\mathrm{C} 11$ & $119.68(15)$ \\
\hline $\mathrm{N} 2-\mathrm{N} 3-\mathrm{C} 10$ & $103.74(13)$ & $\mathrm{C} 13-\mathrm{C} 12-\mathrm{H} 12$ & 120.2 \\
\hline $\mathrm{C} 13-\mathrm{O} 1-\mathrm{C} 17$ & $117.13(13)$ & $\mathrm{C} 11-\mathrm{C} 12-\mathrm{H} 12$ & 120.2 \\
\hline $\mathrm{C} 14-\mathrm{O} 2-\mathrm{C} 18$ & $113.13(13)$ & $\mathrm{O} 1-\mathrm{C} 13-\mathrm{C} 12$ & $124.98(14)$ \\
\hline $\mathrm{C} 15-\mathrm{O} 3-\mathrm{C} 19$ & $116.85(12)$ & $\mathrm{O} 1-\mathrm{C} 13-\mathrm{C} 14$ & $114.89(14)$ \\
\hline $\mathrm{C} 2-\mathrm{C} 1-\mathrm{C} 9$ & $129.19(15)$ & $\mathrm{C} 12-\mathrm{C} 13-\mathrm{C} 14$ & $120.12(15)$ \\
\hline $\mathrm{C} 2-\mathrm{C} 1-\mathrm{S} 1$ & $112.12(12)$ & $\mathrm{O} 2-\mathrm{C} 14-\mathrm{C} 15$ & $118.70(14)$ \\
\hline $\mathrm{C} 9-\mathrm{C} 1-\mathrm{S} 1$ & $118.67(12)$ & $\mathrm{O} 2-\mathrm{C} 14-\mathrm{C} 13$ & $121.49(14)$ \\
\hline $\mathrm{C} 1-\mathrm{C} 2-\mathrm{C} 3$ & $113.49(15)$ & $\mathrm{C} 15-\mathrm{C} 14-\mathrm{C} 13$ & $119.72(14)$ \\
\hline $\mathrm{C} 1-\mathrm{C} 2-\mathrm{H} 2$ & 123.3 & $\mathrm{O} 3-\mathrm{C} 15-\mathrm{C} 16$ & $124.11(15)$ \\
\hline $\mathrm{C} 3-\mathrm{C} 2-\mathrm{H} 2$ & 123.3 & $\mathrm{O} 3-\mathrm{C} 15-\mathrm{C} 14$ & $115.35(14)$ \\
\hline $\mathrm{C} 4-\mathrm{C} 3-\mathrm{C} 8$ & $118.91(16)$ & $\mathrm{C} 16-\mathrm{C} 15-\mathrm{C} 14$ & $120.54(14)$ \\
\hline $\mathrm{C} 4-\mathrm{C} 3-\mathrm{C} 2$ & $129.50(16)$ & $\mathrm{C} 15-\mathrm{C} 16-\mathrm{C} 11$ & $119.66(15)$ \\
\hline
\end{tabular}




\begin{tabular}{|c|c|c|c|}
\hline $\mathrm{C} 8-\mathrm{C} 3-\mathrm{C} 2$ & $111.58(15)$ & $\mathrm{C} 15-\mathrm{C} 16-\mathrm{H} 16$ & 120.2 \\
\hline $\mathrm{C} 5-\mathrm{C} 4-\mathrm{C} 3$ & $119.33(18)$ & $\mathrm{C} 11-\mathrm{C} 16-\mathrm{H} 16$ & 120.2 \\
\hline $\mathrm{C} 5-\mathrm{C} 4-\mathrm{H} 4$ & 120.3 & $\mathrm{O} 1-\mathrm{C} 17-\mathrm{H} 17 \mathrm{~A}$ & 109.5 \\
\hline $\mathrm{C} 3-\mathrm{C} 4-\mathrm{H} 4$ & 120.3 & $\mathrm{O} 1-\mathrm{C} 17-\mathrm{H} 17 \mathrm{~B}$ & 109.5 \\
\hline $\mathrm{C} 4-\mathrm{C} 5-\mathrm{C} 6$ & $120.79(17)$ & H17A-C17-H17B & 109.5 \\
\hline $\mathrm{C} 4-\mathrm{C} 5-\mathrm{H} 5$ & 119.6 & $\mathrm{O} 1-\mathrm{C} 17-\mathrm{H} 17 \mathrm{C}$ & 109.5 \\
\hline $\mathrm{C} 6-\mathrm{C} 5-\mathrm{H} 5$ & 119.6 & $\mathrm{H} 17 \mathrm{~A}-\mathrm{C} 17-\mathrm{H} 17 \mathrm{C}$ & 109.5 \\
\hline $\mathrm{C} 7-\mathrm{C} 6-\mathrm{C} 5$ & $121.26(17)$ & $\mathrm{H} 17 \mathrm{~B}-\mathrm{C} 17-\mathrm{H} 17 \mathrm{C}$ & 109.5 \\
\hline $\mathrm{C} 7-\mathrm{C} 6-\mathrm{H} 6$ & 119.4 & $\mathrm{O} 2-\mathrm{C} 18-\mathrm{H} 18 \mathrm{~A}$ & 109.5 \\
\hline $\mathrm{C} 5-\mathrm{C} 6-\mathrm{H} 6$ & 119.4 & $\mathrm{O} 2-\mathrm{C} 18-\mathrm{H} 18 \mathrm{~B}$ & 109.5 \\
\hline $\mathrm{C} 6-\mathrm{C} 7-\mathrm{C} 8$ & $118.28(18)$ & $\mathrm{H} 18 \mathrm{~A}-\mathrm{C} 18-\mathrm{H} 18 \mathrm{~B}$ & 109.5 \\
\hline $\mathrm{C} 6-\mathrm{C} 7-\mathrm{H} 7$ & 120.9 & $\mathrm{O} 2-\mathrm{C} 18-\mathrm{H} 18 \mathrm{C}$ & 109.5 \\
\hline $\mathrm{C} 8-\mathrm{C} 7-\mathrm{H} 7$ & 120.9 & $\mathrm{H} 18 \mathrm{~A}-\mathrm{C} 18-\mathrm{H} 18 \mathrm{C}$ & 109.5 \\
\hline $\mathrm{C} 7-\mathrm{C} 8-\mathrm{C} 3$ & $121.41(16)$ & $\mathrm{H} 18 \mathrm{~B}-\mathrm{C} 18-\mathrm{H} 18 \mathrm{C}$ & 109.5 \\
\hline $\mathrm{C} 7-\mathrm{C} 8-\mathrm{S} 1$ & $127.30(15)$ & $\mathrm{O} 3-\mathrm{C} 19-\mathrm{H} 19 \mathrm{~A}$ & 109.5 \\
\hline $\mathrm{C} 3-\mathrm{C} 8-\mathrm{S} 1$ & $111.27(12)$ & $\mathrm{O} 3-\mathrm{C} 19-\mathrm{H} 19 \mathrm{~B}$ & 109.5 \\
\hline $\mathrm{N} 1-\mathrm{C} 9-\mathrm{C} 10$ & $108.37(14)$ & H19A-C19-H19B & 109.5 \\
\hline $\mathrm{N} 1-\mathrm{C} 9-\mathrm{C} 1$ & $119.98(15)$ & $\mathrm{O} 3-\mathrm{C} 19-\mathrm{H} 19 \mathrm{C}$ & 109.5 \\
\hline $\mathrm{C} 10-\mathrm{C} 9-\mathrm{C} 1$ & $131.64(14)$ & $\mathrm{H} 19 \mathrm{~A}-\mathrm{C} 19-\mathrm{H} 19 \mathrm{C}$ & 109.5 \\
\hline $\mathrm{N} 3-\mathrm{C} 10-\mathrm{C} 9$ & $107.94(14)$ & $\mathrm{H} 19 \mathrm{~B}-\mathrm{C} 19-\mathrm{H} 19 \mathrm{C}$ & 109.5 \\
\hline $\mathrm{N} 3-\mathrm{C} 10-\mathrm{C} 11$ & $120.88(14)$ & & \\
\hline $\mathrm{C} 9-\mathrm{N} 1-\mathrm{N} 2-\mathrm{N} 3$ & $0.50(19)$ & $\mathrm{C} 1-\mathrm{C} 9-\mathrm{C} 10-\mathrm{N} 3$ & $179.11(17)$ \\
\hline $\mathrm{N} 1-\mathrm{N} 2-\mathrm{N} 3-\mathrm{C} 10$ & $-0.27(19)$ & $\mathrm{N} 1-\mathrm{C} 9-\mathrm{C} 10-\mathrm{C} 11$ & $-177.91(16)$ \\
\hline $\mathrm{C} 8-\mathrm{S} 1-\mathrm{C} 1-\mathrm{C} 2$ & $-1.94(14)$ & $\mathrm{C} 1-\mathrm{C} 9-\mathrm{C} 10-\mathrm{C} 11$ & $0.8(3)$ \\
\hline $\mathrm{C} 8-\mathrm{S} 1-\mathrm{C} 1-\mathrm{C} 9$ & $176.59(14)$ & $\mathrm{N} 3-\mathrm{C} 10-\mathrm{C} 11-\mathrm{C} 16$ & $-148.68(16)$ \\
\hline $\mathrm{C} 9-\mathrm{C} 1-\mathrm{C} 2-\mathrm{C} 3$ & $-176.73(16)$ & $\mathrm{C} 9-\mathrm{C} 10-\mathrm{C} 11-\mathrm{C} 16$ & $29.4(3)$ \\
\hline $\mathrm{S} 1-\mathrm{C} 1-\mathrm{C} 2-\mathrm{C} 3$ & $1.60(19)$ & $\mathrm{N} 3-\mathrm{C} 10-\mathrm{C} 11-\mathrm{C} 12$ & $28.3(2)$ \\
\hline $\mathrm{C} 1-\mathrm{C} 2-\mathrm{C} 3-\mathrm{C} 4$ & $178.36(18)$ & $\mathrm{C} 9-\mathrm{C} 10-\mathrm{C} 11-\mathrm{C} 12$ & $-153.60(18)$ \\
\hline $\mathrm{C} 1-\mathrm{C} 2-\mathrm{C} 3-\mathrm{C} 8$ & $-0.3(2)$ & $\mathrm{C} 16-\mathrm{C} 11-\mathrm{C} 12-\mathrm{C} 13$ & $0.3(2)$ \\
\hline $\mathrm{C} 8-\mathrm{C} 3-\mathrm{C} 4-\mathrm{C} 5$ & $1.1(3)$ & $\mathrm{C} 10-\mathrm{C} 11-\mathrm{C} 12-\mathrm{C} 13$ & $-176.69(15)$ \\
\hline $\mathrm{C} 2-\mathrm{C} 3-\mathrm{C} 4-\mathrm{C} 5$ & $-177.44(17)$ & $\mathrm{C} 17-\mathrm{O} 1-\mathrm{C} 13-\mathrm{C} 12$ & $-1.4(2)$ \\
\hline $\mathrm{C} 3-\mathrm{C} 4-\mathrm{C} 5-\mathrm{C} 6$ & $0.2(3)$ & $\mathrm{C} 17-\mathrm{O} 1-\mathrm{C} 13-\mathrm{C} 14$ & $178.07(15)$ \\
\hline $\mathrm{C} 4-\mathrm{C} 5-\mathrm{C} 6-\mathrm{C} 7$ & $-1.2(3)$ & $\mathrm{C} 11-\mathrm{C} 12-\mathrm{C} 13-\mathrm{O} 1$ & $176.42(15)$ \\
\hline $\mathrm{C} 5-\mathrm{C} 6-\mathrm{C} 7-\mathrm{C} 8$ & $1.0(3)$ & $\mathrm{C} 11-\mathrm{C} 12-\mathrm{C} 13-\mathrm{C} 14$ & $-3.1(2)$ \\
\hline $\mathrm{C} 6-\mathrm{C} 7-\mathrm{C} 8-\mathrm{C} 3$ & $0.3(3)$ & $\mathrm{C} 18-\mathrm{O} 2-\mathrm{C} 14-\mathrm{C} 15$ & $-105.70(17)$ \\
\hline $\mathrm{C} 6-\mathrm{C} 7-\mathrm{C} 8-\mathrm{S} 1$ & $178.72(15)$ & $\mathrm{C} 18-\mathrm{O} 2-\mathrm{C} 14-\mathrm{C} 13$ & $77.81(19)$ \\
\hline $\mathrm{C} 4-\mathrm{C} 3-\mathrm{C} 8-\mathrm{C} 7$ & $-1.4(3)$ & $\mathrm{O} 1-\mathrm{C} 13-\mathrm{C} 14-\mathrm{O} 2$ & $-0.5(2)$ \\
\hline $\mathrm{C} 2-\mathrm{C} 3-\mathrm{C} 8-\mathrm{C} 7$ & $177.41(16)$ & $\mathrm{C} 12-\mathrm{C} 13-\mathrm{C} 14-\mathrm{O} 2$ & $179.04(15)$ \\
\hline $\mathrm{C} 4-\mathrm{C} 3-\mathrm{C} 8-\mathrm{S} 1$ & $-179.98(13)$ & $\mathrm{O} 1-\mathrm{C} 13-\mathrm{C} 14-\mathrm{C} 15$ & $-176.96(14)$ \\
\hline $\mathrm{C} 2-\mathrm{C} 3-\mathrm{C} 8-\mathrm{S} 1$ & $-1.20(19)$ & $\mathrm{C} 12-\mathrm{C} 13-\mathrm{C} 14-\mathrm{C} 15$ & $2.6(2)$ \\
\hline $\mathrm{C} 1-\mathrm{S} 1-\mathrm{C} 8-\mathrm{C} 7$ & $-176.74(18)$ & $\mathrm{C} 19-\mathrm{O} 3-\mathrm{C} 15-\mathrm{C} 16$ & $-10.7(2)$ \\
\hline $\mathrm{C} 1-\mathrm{S} 1-\mathrm{C} 8-\mathrm{C} 3$ & $1.76(14)$ & $\mathrm{C} 19-\mathrm{O} 3-\mathrm{C} 15-\mathrm{C} 14$ & $170.44(14)$ \\
\hline $\mathrm{N} 2-\mathrm{N} 1-\mathrm{C} 9-\mathrm{C} 10$ & $-0.50(18)$ & $\mathrm{O} 2-\mathrm{C} 14-\mathrm{C} 15-\mathrm{O} 3$ & $3.1(2)$ \\
\hline $\mathrm{N} 2-\mathrm{N} 1-\mathrm{C} 9-\mathrm{C} 1$ & $-179.41(15)$ & $\mathrm{C} 13-\mathrm{C} 14-\mathrm{C} 15-\mathrm{O} 3$ & $179.67(15)$ \\
\hline $\mathrm{C} 2-\mathrm{C} 1-\mathrm{C} 9-\mathrm{N} 1$ & $-150.00(18)$ & $\mathrm{O} 2-\mathrm{C} 14-\mathrm{C} 15-\mathrm{C} 16$ & $-175.80(15)$ \\
\hline $\mathrm{S} 1-\mathrm{C} 1-\mathrm{C} 9-\mathrm{N} 1$ & $31.8(2)$ & $\mathrm{C} 13-\mathrm{C} 14-\mathrm{C} 15-\mathrm{C} 16$ & $0.8(2)$ \\
\hline $\mathrm{C} 2-\mathrm{C} 1-\mathrm{C} 9-\mathrm{C} 10$ & $31.4(3)$ & $\mathrm{O} 3-\mathrm{C} 15-\mathrm{C} 16-\mathrm{C} 11$ & $177.62(15)$ \\
\hline
\end{tabular}




\begin{tabular}{llll}
$\mathrm{S} 1-\mathrm{C} 1-\mathrm{C} 9-\mathrm{C} 10$ & $-146.86(16)$ & $\mathrm{C} 14-\mathrm{C} 15-\mathrm{C} 16-\mathrm{C} 11$ & $-3.6(2)$ \\
$\mathrm{N} 2-\mathrm{N} 3-\mathrm{C} 10-\mathrm{C} 9$ & $-0.08(18)$ & $\mathrm{C} 12-\mathrm{C} 11-\mathrm{C} 16-\mathrm{C} 15$ & $3.1(2)$ \\
$\mathrm{N} 2-\mathrm{N} 3-\mathrm{C} 10-\mathrm{C} 11$ & $178.42(15)$ & $\mathrm{C} 10-\mathrm{C} 11-\mathrm{C} 16-\mathrm{C} 15$ & $-179.95(15)$ \\
$\mathrm{N} 1-\mathrm{C} 9-\mathrm{C} 10-\mathrm{N} 3$ & $0.37(19)$ & & \\
\hline
\end{tabular}

Hydrogen-bond geometry $\left(\AA,{ }^{\circ}\right)$

\begin{tabular}{lllll}
\hline$D-\mathrm{H} \cdots A$ & $D-\mathrm{H}$ & $\mathrm{H} \cdots A$ & $D \cdots A$ & $D-\mathrm{H} \cdots A$ \\
\hline $\mathrm{N} 2-\mathrm{H} 2 N \cdots \mathrm{O} 2^{\mathrm{i}}$ & $0.87(2)$ & $2.16(2)$ & $2.9381(18)$ & $147.6(18)$ \\
$\mathrm{N} 2-\mathrm{H} 2 N \cdots 3^{\mathrm{i}}$ & $0.87(2)$ & $2.20(2)$ & $2.8503(18)$ & $130.8(17)$ \\
\hline
\end{tabular}

Symmetry code: (i) $x-1 / 2,-y+3 / 2, z-1 / 2$.

\section{(II) 4-(Benzo[b]thiophen-2-yl)-2-methyl-5-(3,4,5-trimethoxyphenyl)-2H-1,2,3-triazole}

\section{Crystal data}

$\mathrm{C}_{20} \mathrm{H}_{19} \mathrm{~N}_{3} \mathrm{O}_{3} \mathrm{~S}$

$M_{r}=381.44$

Triclinic, $P \overline{1}$

$a=8.8579(1) \AA$

$b=11.0761(1) \AA$

$c=11.2626(1) \AA$

$\alpha=106.859(4)^{\circ}$

$\beta=111.668(5)^{\circ}$

$\gamma=105.498(4)^{\circ}$

$V=891.51(4) \AA^{3}$

\section{Data collection}

Nonius KappaCCD diffractometer

Radiation source: fine-focus sealed-tube Detector resolution: 9.1 pixels $\mathrm{mm}^{-1}$ $\varphi$ and $\omega$ scans at fixed $\chi=55^{\circ}$

Absorption correction: multi-scan

(SADABS; Sheldrick, 2008a)

$T_{\min }=0.858, T_{\max }=0.962$

\section{Refinement}

Refinement on $F^{2}$

Least-squares matrix: full

$R\left[F^{2}>2 \sigma\left(F^{2}\right)\right]=0.037$

$w R\left(F^{2}\right)=0.096$

$S=1.08$

4097 reflections

276 parameters

161 restraints

Primary atom site location: structure-invariant direct methods
$Z=2$

$F(000)=400$

$D_{\mathrm{x}}=1.421 \mathrm{Mg} \mathrm{m}^{-3}$

Mo $K \alpha$ radiation, $\lambda=0.71073 \AA$

Cell parameters from 4076 reflections

$\theta=1.0-27.5^{\circ}$

$\mu=0.21 \mathrm{~mm}^{-1}$

$T=90 \mathrm{~K}$

Cut block, pale yellow

$0.22 \times 0.20 \times 0.15 \mathrm{~mm}$

36591 measured reflections

4097 independent reflections

3572 reflections with $I>2 \sigma(I)$

$R_{\text {int }}=0.045$

$\theta_{\max }=27.6^{\circ}, \theta_{\min }=2.1^{\circ}$

$h=-11 \rightarrow 11$

$k=-14 \rightarrow 14$

$l=-14 \rightarrow 14$

Secondary atom site location: difference Fourier map

Hydrogen site location: difference Fourier map

H-atom parameters constrained

$w=1 /\left[\sigma^{2}\left(F_{\mathrm{o}}^{2}\right)+(0.0472 P)^{2}+0.4023 P\right]$

where $P=\left(F_{\mathrm{o}}^{2}+2 F_{\mathrm{c}}{ }^{2}\right) / 3$

$(\Delta / \sigma)_{\max }=0.001$

$\Delta \rho_{\max }=0.31 \mathrm{e} \AA^{-3}$

$\Delta \rho_{\min }=-0.28$ e $\AA^{-3}$ 


\section{Special details}

Experimental. The crystal was mounted with polyisobutene oil on the tip of a fine glass fibre, which was fastened in a copper mounting pin with electrical solder. It was placed directly into the cold gas stream of a liquid nitrogen based cryostat, according to published methods (Hope, 1994; Parkin \& Hope, 1998).

Diffraction data were collected with the crystal at $90 \mathrm{~K}$, which is standard practice in this laboratory for the majority of flash-cooled crystals.

Geometry. All e.s.d.'s (except the e.s.d. in the dihedral angle between two 1.s. planes) are estimated using the full covariance matrix. The cell e.s.d.'s are taken into account individually in the estimation of e.s.d.'s in distances, angles and torsion angles; correlations between e.s.d.'s in cell parameters are only used when they are defined by crystal symmetry. An approximate (isotropic) treatment of cell e.s.d.'s is used for estimating e.s.d.'s involving 1.s. planes.

Refinement. Refinement progress was checked using PLATON (Spek, 2009) and by an R-tensor (Parkin, 2000). The final model was further checked with the IUCr utility checkCIF.

Fractional atomic coordinates and isotropic or equivalent isotropic displacement parameters $\left(\AA^{2}\right)$

\begin{tabular}{|c|c|c|c|c|c|}
\hline & $x$ & $y$ & $z$ & $U_{\text {iso }} * / U_{\text {eq }}$ & Occ. $(<1)$ \\
\hline N1 & $0.41837(14)$ & $0.62237(11)$ & $0.61716(11)$ & $0.0160(2)$ & \\
\hline $\mathrm{N} 2$ & $0.56418(15)$ & $0.73837(11)$ & $0.71234(11)$ & $0.0158(2)$ & \\
\hline N3 & $0.65185(15)$ & 0.80574 (11) & $0.66113(11)$ & $0.0163(2)$ & \\
\hline $\mathrm{O} 1$ & $0.75768(14)$ & $1.08896(10)$ & $0.37490(10)$ & $0.0234(2)$ & \\
\hline $\mathrm{O} 2$ & $0.77188(12)$ & $0.90655(10)$ & $0.16639(9)$ & $0.0178(2)$ & \\
\hline $\mathrm{O} 3$ & $0.72458(13)$ & $0.64975(10)$ & $0.14267(10)$ & $0.0197(2)$ & \\
\hline $\mathrm{C} 1$ & $0.2506(5)$ & $0.5036(4)$ & $0.36036(19)$ & $0.0145(4)$ & $0.9363(14)$ \\
\hline S1 & $0.08551(5)$ & $0.39081(4)$ & $0.37446(4)$ & $0.01741(12)$ & $0.9363(14)$ \\
\hline $\mathrm{C} 2$ & $0.2034(2)$ & $0.48053(18)$ & $0.22483(17)$ & $0.0176(3)$ & $0.9363(14)$ \\
\hline $\mathrm{H} 2$ & 0.2776 & 0.5336 & 0.1986 & $0.021 *$ & $0.9363(14)$ \\
\hline $\mathrm{C} 3$ & $0.0313(3)$ & $0.3687(3)$ & $0.1242(2)$ & $0.0164(4)$ & $0.9363(14)$ \\
\hline $\mathrm{C} 4$ & $-0.0638(3)$ & $0.31782(16)$ & $-0.0245(2)$ & $0.0203(4)$ & $0.9363(14)$ \\
\hline $\mathrm{H} 4$ & -0.0117 & 0.3565 & -0.0725 & $0.024^{*}$ & 0.9363 (14) \\
\hline $\mathrm{C} 5$ & $-0.2341(3)$ & $0.21078(18)$ & $-0.09952(19)$ & $0.0210(4)$ & $0.9363(14)$ \\
\hline H5 & -0.2990 & 0.1766 & -0.1996 & $0.025^{*}$ & $0.9363(14)$ \\
\hline C6 & $-0.3125(2)$ & $0.1519(2)$ & $-0.03024(16)$ & $0.0195(4)$ & $0.9363(14)$ \\
\hline H6 & -0.4295 & 0.0784 & -0.0841 & $0.023^{*}$ & $0.9363(14)$ \\
\hline $\mathrm{C} 7$ & $-0.2218(2)$ & $0.19944(19)$ & $0.11543(18)$ & $0.0186(4)$ & $0.9363(14)$ \\
\hline $\mathrm{H} 7$ & -0.2742 & 0.1591 & 0.1624 & $0.022 *$ & 0.9363 (14) \\
\hline $\mathrm{C} 8$ & $-0.0508(2)$ & $0.3086(2)$ & $0.19147(18)$ & $0.0161(3)$ & $0.9363(14)$ \\
\hline $\mathrm{C} 1^{\prime}$ & $0.269(8)$ & $0.522(7)$ & $0.368(2)$ & 0.0145 (4) & $0.0637(14)$ \\
\hline $\mathrm{S} 1^{\prime}$ & $0.2341(9)$ & $0.5088(7)$ & $0.2013(7)$ & $0.0176(3)$ & $0.0637(14)$ \\
\hline $\mathrm{C} 2^{\prime}$ & $0.134(3)$ & $0.418(2)$ & 0.3485 (19) & $0.01741(12)$ & $0.0637(14)$ \\
\hline $\mathrm{H} 2^{\prime}$ & 0.1347 & 0.3980 & 0.4251 & $0.021^{*}$ & $0.0637(14)$ \\
\hline $\mathrm{C} 3^{\prime}$ & -0.013 & $0.335(4)$ & $0.207(2)$ & 0.0161 (3) & $0.0637(14)$ \\
\hline $\mathrm{C} 4^{\prime}$ & -0.173 & $0.221(3)$ & 0.150 & $0.0186(4)$ & $0.0637(14)$ \\
\hline $\mathrm{H} 4^{\prime}$ & -0.1949 & 0.1828 & 0.2106 & $0.022 *$ & $0.0637(14)$ \\
\hline $\mathrm{C} 5^{\prime}$ & -0.297 (4) & $0.162(3)$ & 0.013 & 0.0195 (4) & $0.0637(14)$ \\
\hline $\mathrm{H} 5^{\prime}$ & -0.4184 & 0.1070 & -0.0167 & $0.023^{*}$ & $0.0637(14)$ \\
\hline $\mathrm{C} 6^{\prime}$ & $-0.248(4)$ & 0.183 & $-0.086(3)$ & $0.0210(4)$ & 0.0637 (14) \\
\hline $\mathrm{H} 6^{\prime}$ & -0.3283 & 0.1303 & -0.1851 & $0.025^{*}$ & $0.0637(14)$ \\
\hline $\mathrm{C} 7^{\prime}$ & -0.079 (4) & $0.284(3)$ & $-0.036(3)$ & $0.0203(4)$ & $0.0637(14)$ \\
\hline $\mathrm{H} 7^{\prime}$ & -0.0314 & 0.2892 & -0.0985 & $0.024 *$ & $0.0637(14)$ \\
\hline
\end{tabular}




$\begin{array}{lllll}\text { C8 }^{\prime} & 0.020(4) & 0.377(5) & 0.108(2) & 0.0164(4) \\ \text { C9 } & 0.40890(17) & 0.61277(13) & 0.49233(13) & 0.0147(2) \\ \text { C10 } & 0.55623(17) & 0.72729(13) & 0.52005(13) & 0.0146(2) \\ \text { C11 } & 0.61431(17) & 0.77268(13) & 0.42743(13) & 0.0152(3) \\ \text { C12 } & 0.65731(17) & 0.91063(13) & 0.44855(13) & 0.0166(3) \\ \text { H12 } & 0.6472 & 0.9730 & 0.5204 & 0.020^{*} \\ \text { C13 } & 0.71519(17) & 0.95607(13) & 0.36342(14) & 0.0168(3) \\ \text { C14 } & 0.72907(16) & 0.86471(13) & 0.25757(13) & 0.0152(3) \\ \text { C15 } & 0.69315(17) & 0.72810(13) & 0.24151(13) & 0.0157(3) \\ \text { C16 } & 0.63395(17) & 0.68128(13) & 0.32534(13) & 0.0158(3) \\ \text { H16 } & 0.6073 & 0.5881 & 0.3131 & 0.019^{*} \\ \text { C17 } & 0.8251(2) & 1.19553(14) & 0.51199(15) & 0.0231(3) \\ \text { H17A } & 0.7287 & 1.1871 & 0.5366 & 0.035^{*} \\ \text { H17B } & 0.8707 & 1.2866 & 0.5122 & 0.035^{*} \\ \text { H17C } & 0.9222 & 1.1864 & 0.5817 & 0.035^{*} \\ \text { C18 } & 0.96108(18) & 0.98269(14) & 0.22249(14) & 0.0202(3) \\ \text { H18A } & 1.0093 & 1.0624 & 0.3134 & 0.030^{*} \\ \text { H18B } & 0.9813 & 1.0158 & 0.1555 & 0.030^{*} \\ \text { H18C } & 1.0214 & 0.9217 & 0.2368 & 0.030^{*} \\ \text { C19 } & 0.7008(2) & 0.51278(14) & 0.13104(16) & 0.0231(3) \\ \text { H19A } & 0.7781 & 0.5191 & 0.2233 & 0.035^{*} \\ \text { H19B } & 0.7324 & 0.4680 & 0.0608 & 0.035^{*} \\ \text { H19C } & 0.5754 & 0.4577 & 0.1010 & 0.035^{*} \\ \text { C20 } & 0.63205(18) & 0.78558(14) & 0.86407(13) & 0.0185(3) \\ \text { H20A } & 0.7016 & 0.7356 & 0.8977 & 0.028^{*} \\ \text { H20B } & 0.5314 & 0.7673 & 0.8838 & 0.028^{*} \\ \text { H20C } & 0.7089 & 0.8856 & 0.9131 & 0.028^{*}\end{array}$

Atomic displacement parameters $\left(\AA^{2}\right)$

\begin{tabular}{lllllll}
\hline & $U^{11}$ & $U^{22}$ & $U^{33}$ & $U^{12}$ & $U^{13}$ & $U^{23}$ \\
\hline $\mathrm{N} 1$ & $0.0151(5)$ & $0.0152(5)$ & $0.0154(5)$ & $0.0047(4)$ & $0.0069(4)$ & $0.0059(4)$ \\
$\mathrm{N} 2$ & $0.0165(5)$ & $0.0154(5)$ & $0.0138(5)$ & $0.0042(4)$ & $0.0078(4)$ & $0.0059(4)$ \\
$\mathrm{N} 3$ & $0.0167(5)$ & $0.0171(5)$ & $0.0156(5)$ & $0.0054(4)$ & $0.0087(4)$ & $0.0080(4)$ \\
$\mathrm{O} 1$ & $0.0339(6)$ & $0.0148(5)$ & $0.0214(5)$ & $0.0074(4)$ & $0.0139(4)$ & $0.0095(4)$ \\
$\mathrm{O} 2$ & $0.0152(5)$ & $0.0207(5)$ & $0.0139(4)$ & $0.0021(4)$ & $0.0061(4)$ & $0.0093(4)$ \\
$\mathrm{O} 3$ & $0.0235(5)$ & $0.0176(5)$ & $0.0206(5)$ & $0.0070(4)$ & $0.0147(4)$ & $0.0077(4)$ \\
$\mathrm{C} 1$ & $0.0134(11)$ & $0.0112(15)$ & $0.0170(6)$ & $0.0034(6)$ & $0.0072(6)$ & $0.0057(6)$ \\
$\mathrm{S} 1$ & $0.01419(19)$ & $0.01807(19)$ & $0.01687(18)$ & $0.00243(14)$ & $0.00642(14)$ & $0.00913(14)$ \\
$\mathrm{C} 2$ & $0.0158(7)$ & $0.0180(8)$ & $0.0178(7)$ & $0.0032(6)$ & $0.0091(6)$ & $0.0086(6)$ \\
$\mathrm{C} 3$ & $0.0152(7)$ & $0.0161(7)$ & $0.0178(7)$ & $0.0070(6)$ & $0.0078(6)$ & $0.0069(6)$ \\
$\mathrm{C} 4$ & $0.0197(8)$ & $0.0183(10)$ & $0.0186(7)$ & $0.0045(8)$ & $0.0098(6)$ & $0.0051(7)$ \\
$\mathrm{C} 5$ & $0.0192(7)$ & $0.0194(9)$ & $0.0169(7)$ & $0.0056(7)$ & $0.0054(6)$ & $0.0051(6)$ \\
$\mathrm{C} 6$ & $0.0139(7)$ & $0.0159(7)$ & $0.0197(9)$ & $0.0032(5)$ & $0.0030(7)$ & $0.0059(8)$ \\
$\mathrm{C} 7$ & $0.0123(8)$ & $0.0167(8)$ & $0.0219(10)$ & $0.0039(7)$ & $0.0043(7)$ & $0.0096(8)$ \\
$\mathrm{C} 8$ & $0.0138(10)$ & $0.0135(11)$ & $0.0179(7)$ & $0.0048(7)$ & $0.0057(6)$ & $0.0062(7)$ \\
C1 & $0.0134(11)$ & $0.0112(15)$ & $0.0170(6)$ & $0.0034(6)$ & $0.0072(6)$ & $0.0057(6)$ \\
S1 $^{\prime}$ & $0.0158(7)$ & $0.0180(8)$ & $0.0178(7)$ & $0.0032(6)$ & $0.0091(6)$ & $0.0086(6)$
\end{tabular}


supporting information

\begin{tabular}{lllllll}
$\mathrm{C}^{\prime}$ & $0.01419(19)$ & $0.01807(19)$ & $0.01687(18)$ & $0.00243(14)$ & $0.00642(14)$ & $0.00913(14)$ \\
$\mathrm{C}^{\prime}$ & $0.0138(10)$ & $0.0135(11)$ & $0.0179(7)$ & $0.0048(7)$ & $0.0057(6)$ & $0.0062(7)$ \\
$\mathrm{C}^{\prime}$ & $0.0123(8)$ & $0.0167(8)$ & $0.0219(10)$ & $0.0039(7)$ & $0.0043(7)$ & $0.0096(8)$ \\
$\mathrm{C}^{\prime}$ & $0.0139(7)$ & $0.0159(7)$ & $0.0197(9)$ & $0.0032(5)$ & $0.0030(7)$ & $0.0059(8)$ \\
$\mathrm{C}^{\prime}$ & $0.0192(7)$ & $0.0194(9)$ & $0.0169(7)$ & $0.0056(7)$ & $0.0054(6)$ & $0.0051(6)$ \\
$\mathrm{C}^{\prime}$ & $0.0197(8)$ & $0.0183(10)$ & $0.0186(7)$ & $0.0045(8)$ & $0.0098(6)$ & $0.0051(7)$ \\
$\mathrm{C} 8^{\prime}$ & $0.0152(7)$ & $0.0161(7)$ & $0.0178(7)$ & $0.0070(6)$ & $0.0078(6)$ & $0.0069(6)$ \\
$\mathrm{C} 9$ & $0.0155(6)$ & $0.0149(6)$ & $0.0143(6)$ & $0.0065(5)$ & $0.0072(5)$ & $0.0073(5)$ \\
$\mathrm{C} 10$ & $0.0139(6)$ & $0.0148(6)$ & $0.0145(6)$ & $0.0059(5)$ & $0.0067(5)$ & $0.0062(5)$ \\
$\mathrm{C} 11$ & $0.0129(6)$ & $0.0168(6)$ & $0.0139(6)$ & $0.0042(5)$ & $0.0055(5)$ & $0.0074(5)$ \\
$\mathrm{C} 12$ & $0.0168(6)$ & $0.0161(6)$ & $0.0143(6)$ & $0.0053(5)$ & $0.0070(5)$ & $0.0057(5)$ \\
$\mathrm{C} 13$ & $0.0161(6)$ & $0.0145(6)$ & $0.0161(6)$ & $0.0038(5)$ & $0.0055(5)$ & $0.0075(5)$ \\
$\mathrm{C} 14$ & $0.0122(6)$ & $0.0179(6)$ & $0.0123(6)$ & $0.0027(5)$ & $0.0045(5)$ & $0.0076(5)$ \\
$\mathrm{C} 15$ & $0.0126(6)$ & $0.0171(6)$ & $0.0129(6)$ & $0.0039(5)$ & $0.0049(5)$ & $0.0048(5)$ \\
$\mathrm{C} 16$ & $0.0144(6)$ & $0.0149(6)$ & $0.0162(6)$ & $0.0040(5)$ & $0.0068(5)$ & $0.0070(5)$ \\
$\mathrm{C} 17$ & $0.0271(7)$ & $0.0144(6)$ & $0.0252(7)$ & $0.0079(6)$ & $0.0121(6)$ & $0.0066(6)$ \\
$\mathrm{C} 18$ & $0.0161(6)$ & $0.0214(7)$ & $0.0201(7)$ & $0.0034(5)$ & $0.0086(5)$ & $0.0094(6)$ \\
$\mathrm{C} 19$ & $0.0295(8)$ & $0.0184(7)$ & $0.0257(7)$ & $0.0106(6)$ & $0.0178(6)$ & $0.0085(6)$ \\
$\mathrm{C} 20$ & $0.0225(7)$ & $0.0193(6)$ & $0.0126(6)$ & $0.0070(5)$ & $0.0089(5)$ & $0.0063(5)$ \\
& & & & & & \\
\hline
\end{tabular}

Geometric parameters $\left(\stackrel{\AA}{ }{ }^{\circ}\right)$

\begin{tabular}{llll}
\hline $\mathrm{N} 1-\mathrm{N} 2$ & $1.3266(15)$ & $\mathrm{C}^{\prime}-\mathrm{C} 4^{\prime}$ & $1.383(17)$ \\
$\mathrm{N} 1-\mathrm{C} 9$ & $1.3477(16)$ & $\mathrm{C}^{\prime}-\mathrm{C} 8^{\prime}$ & $1.413(17)$ \\
$\mathrm{N} 2-\mathrm{N} 3$ & $1.3279(15)$ & $\mathrm{C}^{\prime}-\mathrm{C} 5^{\prime}$ & $1.346(17)$ \\
$\mathrm{N} 2-\mathrm{C} 20$ & $1.4527(16)$ & $\mathrm{C}^{\prime}-\mathrm{H}^{\prime}$ & 0.9500 \\
$\mathrm{~N} 3-\mathrm{C} 10$ & $1.3450(16)$ & $\mathrm{C}^{\prime}-\mathrm{C} 6^{\prime}$ & $1.393(18)$ \\
$\mathrm{O} 1-\mathrm{C} 13$ & $1.3720(15)$ & $\mathrm{C}^{\prime}-\mathrm{H}^{\prime}$ & 0.9500 \\
$\mathrm{O} 1-\mathrm{C} 17$ & $1.4210(17)$ & $\mathrm{C}^{\prime}-\mathrm{C} 7^{\prime}$ & $1.385(18)$ \\
$\mathrm{O} 2-\mathrm{C} 14$ & $1.3721(14)$ & $\mathrm{C} 6^{\prime}-\mathrm{H} 6^{\prime}$ & 0.9500 \\
$\mathrm{O} 2-\mathrm{C} 18$ & $1.4398(16)$ & $\mathrm{C} 7^{\prime}-\mathrm{C} 8^{\prime}$ & $1.405(18)$ \\
$\mathrm{O} 3-\mathrm{C} 15$ & $1.3666(15)$ & $\mathrm{C} 7^{\prime}-\mathrm{H} 7^{\prime}$ & 0.9500 \\
$\mathrm{O} 3-\mathrm{C} 19$ & $1.4340(16)$ & $\mathrm{C} 9-\mathrm{C} 10$ & $1.4122(17)$ \\
$\mathrm{C} 1-\mathrm{C} 2$ & $1.347(3)$ & $\mathrm{C} 10-\mathrm{C} 11$ & $1.4723(17)$ \\
$\mathrm{C} 1-\mathrm{C} 9$ & $1.466(2)$ & $\mathrm{C} 11-\mathrm{C} 16$ & $1.3943(18)$ \\
$\mathrm{C} 1-\mathrm{S} 1$ & $1.742(2)$ & $\mathrm{C} 11-\mathrm{C} 12$ & $1.3956(18)$ \\
$\mathrm{S} 1-\mathrm{C} 8$ & $1.7380(17)$ & $\mathrm{C} 12-\mathrm{C} 13$ & $0.95030(18)$ \\
$\mathrm{C} 2-\mathrm{C} 3$ & $1.429(2)$ & $\mathrm{C} 12-\mathrm{H} 12$ & $1.3911(18)$ \\
$\mathrm{C} 2-\mathrm{H} 2$ & 0.9500 & $\mathrm{C} 13-\mathrm{C} 14$ & $1.4018(18)$ \\
$\mathrm{C} 3-\mathrm{C} 8$ & $1.409(2)$ & $\mathrm{C} 14-\mathrm{C} 15$ & $1.3926(17)$ \\
$\mathrm{C} 3-\mathrm{C} 4$ & $1.410(2)$ & $\mathrm{C} 15-\mathrm{C} 16$ & 0.9500 \\
$\mathrm{C} 4-\mathrm{C} 5$ & $1.384(2)$ & $\mathrm{C} 16-\mathrm{H} 16$ & 0.9800 \\
$\mathrm{C} 4-\mathrm{H} 4$ & 0.9500 & $\mathrm{C} 17-\mathrm{H} 17 \mathrm{~A}$ & 0.9800 \\
$\mathrm{C} 5-\mathrm{C} 6$ & $1.404(2)$ & $\mathrm{C} 17-\mathrm{H} 17 \mathrm{~B}$ & 0.9800 \\
$\mathrm{C} 5-\mathrm{H} 5$ & 0.9500 & $\mathrm{C} 17-\mathrm{H} 17 \mathrm{C}$ & 0.9800 \\
$\mathrm{C} 6-\mathrm{C} 7$ & $1.383(2)$ & $\mathrm{C} 18-\mathrm{H} 18 \mathrm{~A}$ & 0.9800 \\
$\mathrm{C} 6-\mathrm{H} 6$ & 0.9500 & $\mathrm{C} 18-\mathrm{H} 18 \mathrm{~B}$ & 0.9800 \\
$\mathrm{C} 7-\mathrm{C} 8$ & $1.397(2)$ & $\mathrm{C} 18-\mathrm{H} 18 \mathrm{C}$ & \\
& & &
\end{tabular}




\begin{tabular}{|c|c|c|c|}
\hline C7-H7 & 0.9500 & C19-H19A & 0.9800 \\
\hline $\mathrm{C} 1^{\prime}-\mathrm{C} 2^{\prime}$ & 1.318 (19) & C19-H19B & 0.9800 \\
\hline $\mathrm{C} 1^{\prime}-\mathrm{C} 9$ & $1.32(2)$ & C19-H19C & 0.9800 \\
\hline $\mathrm{C} 1^{\prime}-\mathrm{S} 1^{\prime}$ & $1.74(2)$ & $\mathrm{C} 20-\mathrm{H} 20 \mathrm{~A}$ & 0.9800 \\
\hline $\mathrm{S} 1^{\prime}-\mathrm{C} 8^{\prime}$ & $1.731(18)$ & $\mathrm{C} 20-\mathrm{H} 20 \mathrm{~B}$ & 0.9800 \\
\hline $\mathrm{C} 2^{\prime}-\mathrm{C} 3^{\prime}$ & $1.439(17)$ & $\mathrm{C} 20-\mathrm{H} 20 \mathrm{C}$ & 0.9800 \\
\hline $\mathrm{C} 2^{\prime}-\mathrm{H} 2^{\prime}$ & 0.9500 & & \\
\hline $\mathrm{N} 2-\mathrm{N} 1-\mathrm{C} 9$ & $103.78(10)$ & $\mathrm{C} 8^{\prime}-\mathrm{C} 7^{\prime}-\mathrm{H} 7^{\prime}$ & 120.8 \\
\hline $\mathrm{N} 1-\mathrm{N} 2-\mathrm{N} 3$ & $115.92(10)$ & $\mathrm{C} 7^{\prime}-\mathrm{C} 8^{\prime}-\mathrm{C} 3^{\prime}$ & $119(2)$ \\
\hline $\mathrm{N} 1-\mathrm{N} 2-\mathrm{C} 20$ & $122.69(11)$ & $\mathrm{C} 7^{\prime}-\mathrm{C} 8^{\prime}-\mathrm{S} 1^{\prime}$ & $129(2)$ \\
\hline $\mathrm{N} 3-\mathrm{N} 2-\mathrm{C} 20$ & $121.27(11)$ & $\mathrm{C} 3^{\prime}-\mathrm{C} 8^{\prime}-\mathrm{S} 1^{\prime}$ & $106.9(14)$ \\
\hline $\mathrm{N} 2-\mathrm{N} 3-\mathrm{C} 10$ & $104.04(10)$ & $\mathrm{C} 1{ }^{\prime}-\mathrm{C} 9-\mathrm{N} 1$ & $123.7(13)$ \\
\hline $\mathrm{C} 13-\mathrm{O} 1-\mathrm{C} 17$ & $116.24(10)$ & $\mathrm{C} 1{ }^{\prime}-\mathrm{C} 9-\mathrm{C} 10$ & $127.3(11)$ \\
\hline $\mathrm{C} 14-\mathrm{O} 2-\mathrm{C} 18$ & $113.82(10)$ & $\mathrm{N} 1-\mathrm{C} 9-\mathrm{C} 10$ & $108.26(11)$ \\
\hline $\mathrm{C} 15-\mathrm{O} 3-\mathrm{C} 19$ & $116.41(10)$ & $\mathrm{N} 1-\mathrm{C} 9-\mathrm{C} 1$ & $118.94(13)$ \\
\hline $\mathrm{C} 2-\mathrm{C} 1-\mathrm{C} 9$ & 129.94 (17) & $\mathrm{C} 10-\mathrm{C} 9-\mathrm{C} 1$ & $132.41(13)$ \\
\hline $\mathrm{C} 2-\mathrm{C} 1-\mathrm{S} 1$ & $112.33(14)$ & $\mathrm{N} 3-\mathrm{C} 10-\mathrm{C} 9$ & $108.00(11)$ \\
\hline $\mathrm{C} 9-\mathrm{C} 1-\mathrm{S} 1$ & $117.55(13)$ & $\mathrm{N} 3-\mathrm{C} 10-\mathrm{C} 11$ & $119.08(11)$ \\
\hline $\mathrm{C} 8-\mathrm{S} 1-\mathrm{C} 1$ & $91.33(8)$ & $\mathrm{C} 9-\mathrm{C} 10-\mathrm{C} 11$ & $132.90(12)$ \\
\hline $\mathrm{C} 1-\mathrm{C} 2-\mathrm{C} 3$ & $113.73(19)$ & $\mathrm{C} 16-\mathrm{C} 11-\mathrm{C} 12$ & $120.79(11)$ \\
\hline $\mathrm{C} 1-\mathrm{C} 2-\mathrm{H} 2$ & 123.1 & $\mathrm{C} 16-\mathrm{C} 11-\mathrm{C} 10$ & $120.52(11)$ \\
\hline $\mathrm{C} 3-\mathrm{C} 2-\mathrm{H} 2$ & 123.1 & $\mathrm{C} 12-\mathrm{C} 11-\mathrm{C} 10$ & $118.61(11)$ \\
\hline $\mathrm{C} 8-\mathrm{C} 3-\mathrm{C} 4$ & $118.66(16)$ & $\mathrm{C} 13-\mathrm{C} 12-\mathrm{C} 11$ & $119.45(12)$ \\
\hline $\mathrm{C} 8-\mathrm{C} 3-\mathrm{C} 2$ & $111.50(15)$ & $\mathrm{C} 13-\mathrm{C} 12-\mathrm{H} 12$ & 120.3 \\
\hline $\mathrm{C} 4-\mathrm{C} 3-\mathrm{C} 2$ & $129.79(17)$ & $\mathrm{C} 11-\mathrm{C} 12-\mathrm{H} 12$ & 120.3 \\
\hline $\mathrm{C} 5-\mathrm{C} 4-\mathrm{C} 3$ & $119.15(16)$ & $\mathrm{O} 1-\mathrm{C} 13-\mathrm{C} 14$ & $116.02(11)$ \\
\hline $\mathrm{C} 5-\mathrm{C} 4-\mathrm{H} 4$ & 120.4 & $\mathrm{O} 1-\mathrm{C} 13-\mathrm{C} 12$ & $123.56(12)$ \\
\hline $\mathrm{C} 3-\mathrm{C} 4-\mathrm{H} 4$ & 120.4 & $\mathrm{C} 14-\mathrm{C} 13-\mathrm{C} 12$ & $120.40(12)$ \\
\hline $\mathrm{C} 4-\mathrm{C} 5-\mathrm{C} 6$ & $121.14(16)$ & $\mathrm{O} 2-\mathrm{C} 14-\mathrm{C} 13$ & $120.25(11)$ \\
\hline $\mathrm{C} 4-\mathrm{C} 5-\mathrm{H} 5$ & 119.4 & $\mathrm{O} 2-\mathrm{C} 14-\mathrm{C} 15$ & $120.12(11)$ \\
\hline $\mathrm{C} 6-\mathrm{C} 5-\mathrm{H} 5$ & 119.4 & $\mathrm{C} 13-\mathrm{C} 14-\mathrm{C} 15$ & $119.60(11)$ \\
\hline $\mathrm{C} 7-\mathrm{C} 6-\mathrm{C} 5$ & $120.88(16)$ & $\mathrm{O} 3-\mathrm{C} 15-\mathrm{C} 16$ & $124.29(12)$ \\
\hline $\mathrm{C} 7-\mathrm{C} 6-\mathrm{H} 6$ & 119.6 & $\mathrm{O} 3-\mathrm{C} 15-\mathrm{C} 14$ & $115.29(11)$ \\
\hline $\mathrm{C} 5-\mathrm{C} 6-\mathrm{H} 6$ & 119.6 & $\mathrm{C} 16-\mathrm{C} 15-\mathrm{C} 14$ & $120.40(12)$ \\
\hline $\mathrm{C} 6-\mathrm{C} 7-\mathrm{C} 8$ & $118.04(16)$ & $\mathrm{C} 15-\mathrm{C} 16-\mathrm{C} 11$ & $119.24(12)$ \\
\hline $\mathrm{C} 6-\mathrm{C} 7-\mathrm{H} 7$ & 121.0 & $\mathrm{C} 15-\mathrm{C} 16-\mathrm{H} 16$ & 120.4 \\
\hline $\mathrm{C} 8-\mathrm{C} 7-\mathrm{H} 7$ & 121.0 & $\mathrm{C} 11-\mathrm{C} 16-\mathrm{H} 16$ & 120.4 \\
\hline $\mathrm{C} 7-\mathrm{C} 8-\mathrm{C} 3$ & $122.13(15)$ & $\mathrm{O} 1-\mathrm{C} 17-\mathrm{H} 17 \mathrm{~A}$ & 109.5 \\
\hline $\mathrm{C} 7-\mathrm{C} 8-\mathrm{S} 1$ & $126.75(13)$ & $\mathrm{O} 1-\mathrm{C} 17-\mathrm{H} 17 \mathrm{~B}$ & 109.5 \\
\hline $\mathrm{C} 3-\mathrm{C} 8-\mathrm{S} 1$ & $111.11(11)$ & $\mathrm{H} 17 \mathrm{~A}-\mathrm{C} 17-\mathrm{H} 17 \mathrm{~B}$ & 109.5 \\
\hline $\mathrm{C} 2{ }^{\prime}-\mathrm{C} 1^{\prime}-\mathrm{C} 9$ & $124.6(18)$ & $\mathrm{O} 1-\mathrm{C} 17-\mathrm{H} 17 \mathrm{C}$ & 109.5 \\
\hline $\mathrm{C} 2^{\prime}-\mathrm{C} 1^{\prime}-\mathrm{S} 1^{\prime}$ & $107.3(15)$ & $\mathrm{H} 17 \mathrm{~A}-\mathrm{C} 17-\mathrm{H} 17 \mathrm{C}$ & 109.5 \\
\hline $\mathrm{C} 9-\mathrm{C} 1^{\prime}-\mathrm{S} 1^{\prime}$ & $128.0(18)$ & $\mathrm{H} 17 \mathrm{~B}-\mathrm{C} 17-\mathrm{H} 17 \mathrm{C}$ & 109.5 \\
\hline $\mathrm{C} 8^{\prime}-\mathrm{S} 1^{\prime}-\mathrm{C}^{\prime}$ & $95.8(12)$ & $\mathrm{O} 2-\mathrm{C} 18-\mathrm{H} 18 \mathrm{~A}$ & 109.5 \\
\hline $\mathrm{C} 1^{\prime}-\mathrm{C} 2^{\prime}-\mathrm{C} 3^{\prime}$ & $117.3(18)$ & $\mathrm{O} 2-\mathrm{C} 18-\mathrm{H} 18 \mathrm{~B}$ & 109.5 \\
\hline $\mathrm{C} 1^{\prime}-\mathrm{C} 2^{\prime}-\mathrm{H} 2^{\prime}$ & 121.4 & $\mathrm{H} 18 \mathrm{~A}-\mathrm{C} 18-\mathrm{H} 18 \mathrm{~B}$ & 109.5 \\
\hline $\mathrm{C} 3^{\prime}-\mathrm{C} 2^{\prime}-\mathrm{H} 2^{\prime}$ & 121.4 & $\mathrm{O} 2-\mathrm{C} 18-\mathrm{H} 18 \mathrm{C}$ & 109.5 \\
\hline
\end{tabular}




\begin{tabular}{|c|c|c|c|}
\hline $\mathrm{C} 4^{\prime}-\mathrm{C} 3^{\prime}-\mathrm{C} 8^{\prime}$ & $115.8(17)$ & $\mathrm{H} 18 \mathrm{~A}-\mathrm{C} 18-\mathrm{H} 18 \mathrm{C}$ & 109.5 \\
\hline $\mathrm{C} 4^{\prime}-\mathrm{C} 3^{\prime}-\mathrm{C} 2^{\prime}$ & $132(2)$ & $\mathrm{H} 18 \mathrm{~B}-\mathrm{C} 18-\mathrm{H} 18 \mathrm{C}$ & 109.5 \\
\hline $\mathrm{C} 8^{\prime}-\mathrm{C} 3^{\prime}-\mathrm{C} 2^{\prime}$ & $111.8(16)$ & $\mathrm{O} 3-\mathrm{C} 19-\mathrm{H} 19 \mathrm{~A}$ & 109.5 \\
\hline $\mathrm{C} 5^{\prime}-\mathrm{C} 4^{\prime}-\mathrm{C} 3^{\prime}$ & $122(2)$ & $\mathrm{O} 3-\mathrm{C} 19-\mathrm{H} 19 \mathrm{~B}$ & 109.5 \\
\hline $\mathrm{C}^{\prime}-\mathrm{C} 4^{\prime}-\mathrm{H} 4^{\prime}$ & 118.9 & $\mathrm{H} 19 \mathrm{~A}-\mathrm{C} 19-\mathrm{H} 19 \mathrm{~B}$ & 109.5 \\
\hline $\mathrm{C} 3^{\prime}-\mathrm{C} 4^{\prime}-\mathrm{H} 4^{\prime}$ & 118.9 & $\mathrm{O} 3-\mathrm{C} 19-\mathrm{H} 19 \mathrm{C}$ & 109.5 \\
\hline $\mathrm{C} 4^{\prime}-\mathrm{C} 5^{\prime}-\mathrm{C} 6^{\prime}$ & $120(2)$ & $\mathrm{H} 19 \mathrm{~A}-\mathrm{C} 19-\mathrm{H} 19 \mathrm{C}$ & 109.5 \\
\hline $\mathrm{C} 4^{\prime}-\mathrm{C} 5^{\prime}-\mathrm{H} 5^{\prime}$ & 120.2 & $\mathrm{H} 19 \mathrm{~B}-\mathrm{C} 19-\mathrm{H} 19 \mathrm{C}$ & 109.5 \\
\hline $\mathrm{C} 6^{\prime}-\mathrm{C} 5^{\prime}-\mathrm{H} 5^{\prime}$ & 120.2 & $\mathrm{~N} 2-\mathrm{C} 20-\mathrm{H} 20 \mathrm{~A}$ & 109.5 \\
\hline $\mathrm{C} 7^{\prime}-\mathrm{C} 6^{\prime}-\mathrm{C} 5^{\prime}$ & $118(2)$ & $\mathrm{N} 2-\mathrm{C} 20-\mathrm{H} 20 \mathrm{~B}$ & 109.5 \\
\hline $\mathrm{C} 7^{\prime}-\mathrm{C} 6^{\prime}-\mathrm{H} 6^{\prime}$ & 121.0 & $\mathrm{H} 20 \mathrm{~A}-\mathrm{C} 20-\mathrm{H} 20 \mathrm{~B}$ & 109.5 \\
\hline $\mathrm{C} 5^{\prime}-\mathrm{C} 6^{\prime}-\mathrm{H} 6^{\prime}$ & 121.0 & $\mathrm{~N} 2-\mathrm{C} 20-\mathrm{H} 20 \mathrm{C}$ & 109.5 \\
\hline $\mathrm{C} 6^{\prime}-\mathrm{C} 7^{\prime}-\mathrm{C} 8^{\prime}$ & $118(2)$ & $\mathrm{H} 20 \mathrm{~A}-\mathrm{C} 20-\mathrm{H} 20 \mathrm{C}$ & 109.5 \\
\hline $\mathrm{C} 6^{\prime}-\mathrm{C} 7^{\prime}-\mathrm{H} 7^{\prime}$ & 120.8 & $\mathrm{H} 20 \mathrm{~B}-\mathrm{C} 20-\mathrm{H} 20 \mathrm{C}$ & 109.5 \\
\hline $\mathrm{C} 9-\mathrm{N} 1-\mathrm{N} 2-\mathrm{N} 3$ & $-0.21(14)$ & $\mathrm{S} 11^{\prime}-\mathrm{C} 1{ }^{\prime}-\mathrm{C} 9-\mathrm{C} 10$ & $6(12)$ \\
\hline $\mathrm{C} 9-\mathrm{N} 1-\mathrm{N} 2-\mathrm{C} 20$ & $-176.39(11)$ & $\mathrm{S} 11^{\prime}-\mathrm{C} 11^{\prime}-\mathrm{C} 9-\mathrm{C} 1$ & $-157(59)$ \\
\hline $\mathrm{N} 1-\mathrm{N} 2-\mathrm{N} 3-\mathrm{C} 10$ & $-0.26(14)$ & $\mathrm{N} 2-\mathrm{N} 1-\mathrm{C} 9-\mathrm{C}^{\prime}$ & $-170(6)$ \\
\hline $\mathrm{C} 20-\mathrm{N} 2-\mathrm{N} 3-\mathrm{C} 10$ & $175.99(11)$ & $\mathrm{N} 2-\mathrm{N} 1-\mathrm{C} 9-\mathrm{C} 10$ & $0.57(13)$ \\
\hline $\mathrm{C} 2-\mathrm{C} 1-\mathrm{S} 1-\mathrm{C} 8$ & $0.6(4)$ & $\mathrm{N} 2-\mathrm{N} 1-\mathrm{C} 9-\mathrm{C} 1$ & $-173.1(3)$ \\
\hline $\mathrm{C} 9-\mathrm{C} 1-\mathrm{S} 1-\mathrm{C} 8$ & $176.2(4)$ & $\mathrm{S} 1-\mathrm{C} 1-\mathrm{C} 9-\mathrm{C} 1^{\prime}$ & $-154(51)$ \\
\hline $\mathrm{C} 9-\mathrm{C} 1-\mathrm{C} 2-\mathrm{C} 3$ & $-175.7(5)$ & $\mathrm{C} 2-\mathrm{C} 1-\mathrm{C} 9-\mathrm{N} 1$ & $173.7(5)$ \\
\hline $\mathrm{S} 1-\mathrm{C} 1-\mathrm{C} 2-\mathrm{C} 3$ & $-0.7(5)$ & $\mathrm{S} 1-\mathrm{C} 1-\mathrm{C} 9-\mathrm{N} 1$ & $-1.1(5)$ \\
\hline $\mathrm{C} 1-\mathrm{C} 2-\mathrm{C} 3-\mathrm{C} 8$ & $0.5(4)$ & $\mathrm{C} 2-\mathrm{C} 1-\mathrm{C} 9-\mathrm{C} 10$ & $1.8(8)$ \\
\hline $\mathrm{C} 1-\mathrm{C} 2-\mathrm{C} 3-\mathrm{C} 4$ & $177.8(4)$ & $\mathrm{S} 1-\mathrm{C} 1-\mathrm{C} 9-\mathrm{C} 10$ & $-172.95(18)$ \\
\hline $\mathrm{C} 8-\mathrm{C} 3-\mathrm{C} 4-\mathrm{C} 5$ & $0.0(4)$ & $\mathrm{N} 2-\mathrm{N} 3-\mathrm{C} 10-\mathrm{C} 9$ & $0.60(13)$ \\
\hline $\mathrm{C} 2-\mathrm{C} 3-\mathrm{C} 4-\mathrm{C} 5$ & $-177.2(3)$ & $\mathrm{N} 2-\mathrm{N} 3-\mathrm{C} 10-\mathrm{C} 11$ & $179.11(11)$ \\
\hline $\mathrm{C} 3-\mathrm{C} 4-\mathrm{C} 5-\mathrm{C} 6$ & $-0.4(3)$ & $\mathrm{C} 1{ }^{\prime}-\mathrm{C} 9-\mathrm{C} 10-\mathrm{N} 3$ & $170(6)$ \\
\hline $\mathrm{C} 4-\mathrm{C} 5-\mathrm{C} 6-\mathrm{C} 7$ & $0.1(3)$ & $\mathrm{N} 1-\mathrm{C} 9-\mathrm{C} 10-\mathrm{N} 3$ & $-0.76(14)$ \\
\hline $\mathrm{C} 5-\mathrm{C} 6-\mathrm{C} 7-\mathrm{C} 8$ & $0.6(3)$ & $\mathrm{C} 1-\mathrm{C} 9-\mathrm{C} 10-\mathrm{N} 3$ & $171.7(4)$ \\
\hline $\mathrm{C} 6-\mathrm{C} 7-\mathrm{C} 8-\mathrm{C} 3$ & $-1.1(4)$ & $\mathrm{C} 11^{\prime}-\mathrm{C} 9-\mathrm{C} 10-\mathrm{C} 11$ & $-9(6)$ \\
\hline $\mathrm{C} 6-\mathrm{C} 7-\mathrm{C} 8-\mathrm{S} 1$ & $177.16(19)$ & $\mathrm{N} 1-\mathrm{C} 9-\mathrm{C} 10-\mathrm{C} 11$ & $-178.99(13)$ \\
\hline $\mathrm{C} 4-\mathrm{C} 3-\mathrm{C} 8-\mathrm{C} 7$ & $0.8(4)$ & $\mathrm{C} 1-\mathrm{C} 9-\mathrm{C} 10-\mathrm{C} 11$ & $-6.5(4)$ \\
\hline $\mathrm{C} 2-\mathrm{C} 3-\mathrm{C} 8-\mathrm{C} 7$ & $178.4(2)$ & $\mathrm{N} 3-\mathrm{C} 10-\mathrm{C} 11-\mathrm{C} 16$ & $129.29(13)$ \\
\hline $\mathrm{C} 4-\mathrm{C} 3-\mathrm{C} 8-\mathrm{S} 1$ & $-177.7(2)$ & $\mathrm{C} 9-\mathrm{C} 10-\mathrm{C} 11-\mathrm{C} 16$ & $-52.6(2)$ \\
\hline $\mathrm{C} 2-\mathrm{C} 3-\mathrm{C} 8-\mathrm{S} 1$ & $-0.1(3)$ & $\mathrm{N} 3-\mathrm{C} 10-\mathrm{C} 11-\mathrm{C} 12$ & $-47.72(17)$ \\
\hline $\mathrm{C} 1-\mathrm{S} 1-\mathrm{C} 8-\mathrm{C} 7$ & $-178.7(3)$ & $\mathrm{C} 9-\mathrm{C} 10-\mathrm{C} 11-\mathrm{C} 12$ & $130.36(15)$ \\
\hline $\mathrm{C} 1-\mathrm{S} 1-\mathrm{C} 8-\mathrm{C} 3$ & $-0.3(3)$ & $\mathrm{C} 16-\mathrm{C} 11-\mathrm{C} 12-\mathrm{C} 13$ & $2.02(19)$ \\
\hline $\mathrm{C} 2^{\prime}-\mathrm{C} 1^{\prime}-\mathrm{S} 1^{\prime}-\mathrm{C} 8^{\prime}$ & $9(7)$ & $\mathrm{C} 10-\mathrm{C} 11-\mathrm{C} 12-\mathrm{C} 13$ & $179.01(12)$ \\
\hline $\mathrm{C} 9-\mathrm{C} 1^{\prime}-\mathrm{S} 1^{\prime}-\mathrm{C} 8^{\prime}$ & $-176(9)$ & $\mathrm{C} 17-\mathrm{O} 1-\mathrm{C} 13-\mathrm{C} 14$ & $-151.61(12)$ \\
\hline $\mathrm{C} 9-\mathrm{C} 1^{\prime}-\mathrm{C} 2^{\prime}-\mathrm{C} 3^{\prime}$ & $175(7)$ & $\mathrm{C} 17-\mathrm{O} 1-\mathrm{C} 13-\mathrm{C} 12$ & $29.78(18)$ \\
\hline $\mathrm{S} 1^{\prime}-\mathrm{C} 1^{\prime}-\mathrm{C} 2^{\prime}-\mathrm{C} 3^{\prime}$ & $-8(8)$ & $\mathrm{C} 11-\mathrm{C} 12-\mathrm{C} 13-\mathrm{O} 1$ & $178.95(12)$ \\
\hline $\mathrm{C} 1^{\prime}-\mathrm{C} 2^{\prime}-\mathrm{C} 3^{\prime}-\mathrm{C} 4^{\prime}$ & $-180(7)$ & $\mathrm{C} 11-\mathrm{C} 12-\mathrm{C} 13-\mathrm{C} 14$ & $0.40(19)$ \\
\hline $\mathrm{C} 1^{\prime}-\mathrm{C} 2^{\prime}-\mathrm{C} 3^{\prime}-\mathrm{C} 8^{\prime}$ & $4(8)$ & $\mathrm{C} 18-\mathrm{O} 2-\mathrm{C} 14-\mathrm{C} 13$ & $82.87(15)$ \\
\hline $\mathrm{C} 8^{\prime}-\mathrm{C} 3^{\prime}-\mathrm{C} 4^{\prime}-\mathrm{C} 5^{\prime}$ & $-7(7)$ & $\mathrm{C} 18-\mathrm{O} 2-\mathrm{C} 14-\mathrm{C} 15$ & $-99.29(14)$ \\
\hline $\mathrm{C} 2^{\prime}-\mathrm{C} 3^{\prime}-\mathrm{C} 4^{\prime}-\mathrm{C} 5^{\prime}$ & $177(4)$ & $\mathrm{O} 1-\mathrm{C} 13-\mathrm{C} 14-\mathrm{O} 2$ & $-4.02(18)$ \\
\hline $\mathrm{C} 3^{\prime}-\mathrm{C} 4^{\prime}-\mathrm{C} 5^{\prime}-\mathrm{C} 6^{\prime}$ & $21(6)$ & $\mathrm{C} 12-\mathrm{C} 13-\mathrm{C} 14-\mathrm{O} 2$ & $174.63(11)$ \\
\hline $\mathrm{C} 4^{\prime}-\mathrm{C} 5^{\prime}-\mathrm{C} 6^{\prime}-\mathrm{C} 7^{\prime}$ & $-11(6)$ & $\mathrm{O} 1-\mathrm{C} 13-\mathrm{C} 14-\mathrm{C} 15$ & $178.13(11)$ \\
\hline
\end{tabular}




\begin{tabular}{|c|c|c|c|}
\hline $\mathrm{C} 5^{\prime}-\mathrm{C} 6^{\prime}-\mathrm{C} 7^{\prime}-\mathrm{C} 8^{\prime}$ & $-12(7)$ & $\mathrm{C} 12-\mathrm{C} 13-\mathrm{C} 14-\mathrm{C} 15$ & $-3.22(19)$ \\
\hline $\mathrm{C} 6^{\prime}-\mathrm{C} 7^{\prime}-\mathrm{C} 8^{\prime}-\mathrm{C} 3^{\prime}$ & $27(8)$ & $\mathrm{C} 19-\mathrm{O} 3-\mathrm{C} 15-\mathrm{C} 16$ & $-2.60(18)$ \\
\hline $\mathrm{C} 6^{\prime}-\mathrm{C} 7^{\prime}-\mathrm{C} 8^{\prime}-\mathrm{S} 1^{\prime}$ & $178(4)$ & $\mathrm{C} 19-\mathrm{O} 3-\mathrm{C} 15-\mathrm{C} 14$ & $175.84(11)$ \\
\hline $\mathrm{C} 4^{\prime}-\mathrm{C} 3^{\prime}-\mathrm{C} 8^{\prime}-\mathrm{C} 7^{\prime}$ & $-17(8)$ & $\mathrm{O} 2-\mathrm{C} 14-\mathrm{C} 15-\mathrm{O} 3$ & $7.32(17)$ \\
\hline $\mathrm{C} 2^{\prime}-\mathrm{C} 3^{\prime}-\mathrm{C} 8^{\prime}-\mathrm{C} 7^{\prime}$ & $160(5)$ & $\mathrm{C} 13-\mathrm{C} 14-\mathrm{C} 15-\mathrm{O} 3$ & $-174.83(11)$ \\
\hline $\mathrm{C} 4^{\prime}-\mathrm{C} 3^{\prime}-\mathrm{C} 8^{\prime}-\mathrm{S} 1^{\prime}$ & $-174(4)$ & $\mathrm{O} 2-\mathrm{C} 14-\mathrm{C} 15-\mathrm{C} 16$ & $-174.18(11)$ \\
\hline $\mathrm{C} 2^{\prime}-\mathrm{C} 3^{\prime}-\mathrm{C} 8^{\prime}-\mathrm{S} 1^{\prime}$ & $3(6)$ & $\mathrm{C} 13-\mathrm{C} 14-\mathrm{C} 15-\mathrm{C} 16$ & $3.68(19)$ \\
\hline $\mathrm{C} 1^{\prime}-\mathrm{S} 1^{\prime}-\mathrm{C} 8^{\prime}-\mathrm{C} 7^{\prime}$ & $-160(6)$ & $\mathrm{O} 3-\mathrm{C} 15-\mathrm{C} 16-\mathrm{C} 11$ & $177.06(12)$ \\
\hline $\mathrm{C} 1^{\prime}-\mathrm{S} 1^{\prime}-\mathrm{C} 8^{\prime}-\mathrm{C} 3^{\prime}$ & $-6(6)$ & $\mathrm{C} 14-\mathrm{C} 15-\mathrm{C} 16-\mathrm{C} 11$ & $-1.30(19)$ \\
\hline $\mathrm{C} 2^{\prime}-\mathrm{C} 1^{\prime}-\mathrm{C} 9-\mathrm{N} 1$ & $-10(12)$ & $\mathrm{C} 12-\mathrm{C} 11-\mathrm{C} 16-\mathrm{C} 15$ & $-1.56(19)$ \\
\hline $\mathrm{S} 1{ }^{\prime}-\mathrm{C} 1{ }^{\prime}-\mathrm{C} 9-\mathrm{N} 1$ & $174(5)$ & $\mathrm{C} 10-\mathrm{C} 11-\mathrm{C} 16-\mathrm{C} 15$ & $-178.49(12)$ \\
\hline $\mathrm{C} 2^{\prime}-\mathrm{C} 1^{\prime}-\mathrm{C} 9-\mathrm{C} 10$ & $-179(6)$ & & \\
\hline
\end{tabular}

